\title{
ORTHOGONAL CALCULUS
}

\author{
MICHAEL WEISS
}

\begin{abstract}
Orthogonal calculus is a calculus of functors, similar to Goodwillie's calculus. The functors in question take finite dimensional real vector spaces (with an inner product) to pointed spaces. Prime example: $F(V)=B O(V)$, where $O(V)$ is the orthogonal group of $V$. In this example, and in general, first derivatives in the orthogonal calculus reproduce and generalize much of the theory of Stiefel-Whitney classes. Similarly, second derivatives in the orthogonal calculus reproduce and generalize much of the theory of Pontryagin classes.
\end{abstract}

\section{INTRODUCTION}

Let $\mathscr{J}$ be the category of finite dimensional real vector spaces with a positive definite inner product. Morphisms in $\mathscr{J}$ are linear maps respecting the inner product. Orthogonal calculus is concerned with continuous functors $E$ from $\mathcal{J}$ to spaces; continuous means that the evaluation map

$$
\operatorname{mor}(V, W) \times E(V) \longrightarrow E(W)
$$

is continuous, for $V, W$ in $\mathscr{J}$. Here are some easy examples (notation explained further down):

(1) $E(V)=B O(V)$,

(2) $E(V)=B$ TOP $(V)$,

(3) $E(V)=B \mathscr{G}(S(V))$.

Notation: $O(V)$ is the orthogonal group of $V$, and $\operatorname{TOP}(V)$ is the group of homeomorphisms from $V$ to itself. $S(V)$ is the unit sphere in $V$, and $\mathscr{G} S(V)$ is the grouplike monoid of homotopy equivalences from $S(V)$ to itself.

These examples suggest, correctly, that orthogonal calculus is concerned with certain "classical" spaces, such as $B O, B$ TOP,$B \mathscr{G}$, equipped with a sophisticated filtration indexed by the finite dimensional linear subspaces $V$ of $\mathbb{R}^{\infty}$. (There is no loss of generality in allowing only those $V$ wich are contained as linear subspaces in $\mathbb{R}^{\infty}$, but there are some inconveniences.) Other examples of spaces with such a filtration came up in [WW1]. One of the main insights of [WW1] is that much of the theory of Stiefel-Whitney classes (better, the total Stiefel-Whitney class) for $B O$ can be generalised to this setting. I hasten to add that we never put it quite that way in [WW1]. One of the purposes of

Received by the editors November 10, 1993.

1991 Mathematics Subject Classification. Primary 55Q99, 55S99, 55R 40.

Key words and phrases. Calculus of functors, characteristic classes.

Partially supported by the Danish National Science Foundation. 
the present paper is to set up a similar theory of "intrinsic" total Pontryagin classes. Under the influence of Tom Goodwillie, this has turned into a calculus where total Stiefel-Whitney classes appear as first-order approximations, and total Pontryagin classes as second order approximations. Disclaimer: Usually the applications of orthogonal calculus are relative. For example, the orthogonal calculus as such does not really tell us much about $H^{*}(B O ; \mathbb{Z} / 2)$, but it does tell us about $H^{*}(-; \mathbb{Z} / 2)$ of the homotopy fiber of $B O(n) \hookrightarrow B O$, for any $n \geq 0$. In particular, the looped Stiefel-Whitney classes $\Omega w_{k}$ in $H^{k-1}(O ; \mathbb{Z} / 2)$ are repoduced by the orthogonal calculus.

According to Newton and Leibniz, calculus is about derivatives or fluxions: more concretely, about notions like speed, acceleration, and so on. According to Taylor, calculus is also about approximation by polynomials. Let me begin with the Newton-Leibniz point of view.

For a continuous functor $E$ from $\mathscr{J}$ to pointed spaces, define $E^{(1)}$ by

$$
E^{(1)}(V):=\text { hofiber }[E(V) \stackrel{E(l)}{\longrightarrow} E(\mathbb{R} \oplus V)]
$$

where hofiber means homotopy fiber and $l$ is the inclusion of $V$ in $\mathbb{R} \oplus V$. Then $E^{(1)}$, which we may call the derivative of $E$, is again a continuous functor from $\mathcal{J}$ to pointed spaces. Surprisingly, it comes with an additional structure, namely, a binatural transformation

$$
\sigma: V^{c} \wedge E^{(1)}(W) \longrightarrow E^{(1)}(V \oplus W)
$$

where $V^{c}$ denotes the one-point compactification of $V$ with base point $\infty$. For $V=0$ and arbitrary $W$, this agrees with the obvious identification

$$
S^{0} \wedge E^{(1)}(W) \longrightarrow E^{(1)}(W) .
$$

Moreover, $\sigma$ has an associativity property. For a simple example, take the case where $E(V)=B O(V)$; then $E^{(1)}(V) \simeq V^{c}$, and $\sigma$ becomes the homeomorphism

$$
V^{c} \wedge W^{c} \longrightarrow(V \oplus W)^{c} .
$$

Back to the general case: We define the second derivative $E^{(2)}$ by

$$
E^{(2)}(V):=\text { hofiber }\left[\sigma^{a d}: E^{(1)}(V) \rightarrow \Omega E^{(1)}(\mathbb{R} \oplus V)\right] .
$$

where $\sigma^{a d}$ is the adjoint of $\sigma$ in (!). Again this comes with a surprising additional structure: a binatural transformation

$$
\sigma:(2 V)^{c} \wedge E^{(2)}(W) \longrightarrow E^{(2)}(V \oplus W)
$$

where $2 V:=\mathbb{R}^{2} \otimes V$. Again, $\sigma$ agrees with the obious identification when $V=0$, and again, it has an associativity property. We define the third derivative by

$$
E^{(3)}(V):=\text { hofiber }\left[\sigma^{a d}: E^{(2)}(V) \rightarrow \Omega^{2} E^{(2)}(\mathbb{R} \oplus V)\right]
$$

(using the adjoint of $\sigma$ in (!!)), and this comes with

$$
\sigma:(3 V)^{c} \wedge E^{(3)}(W) \longrightarrow E^{(3)}(V \oplus W)
$$

-and so on. See sections 1 and 2 for details and examples.

As a curious by-product, we obtain from $E$ a sequence of spectra. The first of these, denoted $\mathbf{\Theta} E^{(1)}$, has $k$-th term $E^{(1)}\left(\mathbb{R}^{k}\right)$, and the structure maps (from the suspension of the $k$-th term to the $(k+1)$-th term) are specializations of $\sigma$ 
in (!). The second, denoted $\boldsymbol{\Theta} E^{(2)}$, has $2 k$-th term $E^{(2)}\left(\mathbb{R}^{k}\right)$, and the structure maps (from the double suspension of the $2 k$-th term to the $(2 k+2)$-th term) are specializations of $\sigma$ in (!!). And so on. We may think of $\boldsymbol{\theta} E^{(i)}$ as the $i$-th derivative of $E$ at infinity. Orthogonal calculus is very much an attempt to predict the global behaviour of $E$ in terms of these higher derivatives at infinity.

The calculation or identification of $\mathbf{\Theta} E^{(i)}$ (up to homotopy type, say) can be enormously difficult. In the case $E(V)=B O(V)$ one finds

$$
\boldsymbol{\Theta} E^{(1)} \simeq \mathbf{S}^{0}, \quad \boldsymbol{\theta} E^{(2)} \simeq \Omega \mathbf{S}^{0}
$$

(without difficulty), and then, with difficulty,

$$
\boldsymbol{\theta} E^{(3)} \simeq \Omega^{2} \mathbf{m o}(3)
$$

where $\operatorname{mo}(3)$ is a $\mathbb{Z} / 3-$ Moore spectrum. Details can be found in section 2. (The calculation of $\boldsymbol{\theta} E^{(3)}$ is due to Goodwillie, who showed it to me in 1988; later John Klein showed it to me once more. In the meantime, Goodwillie had found something like a closed formula for all the $\boldsymbol{\Theta} E^{(i)}$.) For another example which is closer to my heart, take $E(V)=B \operatorname{TOP}(V)$. Then $\boldsymbol{\Theta} E^{(1)}$ is Waldhausen's $A$-theory of a point,

$$
\boldsymbol{\Theta} E^{(1)} \simeq \mathbf{A}(*) .
$$

(This is clear from [WaMA, Theorem 2, Addendum, (4)].) But $\boldsymbol{\theta} E^{(2)}$ is nameless in this case, and apparently quite hard to understand.

We are now ready for the definition of a polynomial functor. There is one obvious requirement: If $E$ from $\mathscr{J}$ to pointed spaces wants to be called polynomial of degree $\leq n$, then the $(n+1)$-th derivative $E^{(n+1)}$ must vanish (i.e., its values should be contractible spaces). Unfortunately, this is not quite enough, as the case $n=0$ shows: Clearly $E$ should be called polynomial of degree 0 if and only if it takes all morphisms in $\mathcal{J}$ to homotopy equivalences. But a functor with vanishing first derivative need not have this property. The following proposition suggests a solution.

Proposition (5.3). For any continuous $E$ from $\mathcal{J}$ to pointed spaces, and any $n \geq 0$, there is a natural fibration sequence up to homotopy

$$
E^{(n+1)}(V) \stackrel{u}{\longrightarrow} E(V) \stackrel{\rho}{\longrightarrow} \operatorname{holim}_{0 \neq U \subset \mathbb{R}^{n+1}} E(U \oplus V) .
$$

Explanations. The indexing category for the homotopy inverse limit is the partially ordered space of nonzero linear subspaces $U$ of $\mathbb{R}^{n+1}$. This is a topological category, and its topology is invoived in the homotopy inverse limit. The map $\rho$ is obvious, and $u$ is essentially a forgetful map (also fairly obvious from the sketchy description of $\left.E^{(n+1)}\right)$.

Definition (5.1). A continuous $E$ from $\mathscr{J}$ to spaces is polynomial of degree $\leq n$ if

$$
\rho: E(V) \longrightarrow \operatorname{holim}_{0 \neq U \subset \mathbb{R}^{n+1}} E(U \oplus V)
$$

is a homotopy equivalence for all $V$.

Note that the codomain of $\rho$ in this definition is the best guess available about the homotopy type of $E(V)$ if one only knows $E(U \oplus V)$ (and various induced maps) for all nonzero linear subspaces $U$ of $\mathbb{R}^{n+1}$. In other words, 
the defining property is an extrapolation property. Note also the disappearance of base points.

The next goal must be to approximate arbitrary functors, preferably the classical ones, by polynomial functors. To this end we prove, in section 6 , an approximation theorem: for fixed $n \geq 0$, every continuous functor $E$ from $\mathcal{J}$ to spaces has a "universal" approximation

$$
\eta_{n}: E \longrightarrow T_{n} E
$$

by a functor $T_{n} E$ which is polynomial of degree $\leq n$. (Ideally, one would like to say that any other natural transformation from $E$ to a functor which is polynomial of degree $\leq n$ factors uniquely through $\eta_{n}$. However, this is clearly too strong a requirement in a homotopy invariant setting, and the truth about $\eta_{n}$ is more down to earth.) The interest in these approximations stems from the fact that, for $n=1$, they seem to capture intrinsic Stiefel-Whitney type invariants, and for $n=2$, they seem to capture intrinsic Pontryagin type invariants. For the moment, we note that $E$ determines a Taylor tower

$$
\cdots \rightarrow T_{n+1} E \stackrel{r_{n+1}}{\longrightarrow} T_{n} E \stackrel{r_{n}}{\longrightarrow} T_{n-1} E \stackrel{r_{n-1}}{\longrightarrow} \cdots \quad \cdots \stackrel{r_{1}}{\longrightarrow} T_{0} E
$$

where the natural transformations $\left(r_{n}\right)_{n>0}$ satisfy

$$
r_{n} \eta_{n}=\eta_{n-1}: E \longrightarrow T_{n-1} E \text {. }
$$

Existence and uniqueness of the $r_{n}$ "should" follow from the universal property of the $\eta_{n}$ and the (nontrivial) fact that a polynomial functor of degree $\leq n-1$ is also polynomial of degree $\leq n$. But an explicit construction of $r_{n}$ can also be given, and this is fortunate and time-saving in view of the shortcomings of the so-called universal property. In practice, $E$ is a functor from $\mathcal{J}$ to pointed spaces; if this is the case, then $T_{n} E$ has the same property for all $n>0$ and we should ask:

(1) For $V$ in $\mathcal{J}$, what is $\left(T_{0} E\right)(V)$ ?

(2) What is the homotopy fiber of $r_{n}:\left(T_{n} E\right)(V) \rightarrow\left(T_{n-1}\right)(V)$ over the base point?

Answer to question (1):

$$
\left(T_{0} E\right)(V)=\underset{j}{\operatorname{hocolim}} E\left(\mathbb{R}^{j} \oplus V\right) \simeq \underset{j}{\operatorname{hocolim}} E\left(\mathbb{R}^{j}\right)=: E\left(\mathbb{R}^{\infty}\right)
$$

(where the homotopy colimits are just telescopes). The right-hand term $E\left(\mathbb{R}^{\infty}\right)$ is what we may regard as the big and usually classical space under investigation. Answer to question (2) (explanations further down):

Theorem (9.1). The homotopy fiber has the form

$$
\Omega^{\infty}\left[\left((n V)^{c} \wedge \boldsymbol{\theta}\right)_{h O(n)}\right] .
$$

where $\boldsymbol{\Theta} \simeq \boldsymbol{\Theta} E^{(n)}$ is the $n$-th derivative of $E$ at infinity.

Explanation. $\Omega^{\infty}$ is the functor taking a spectrum to its zero-th infinite loop space. The subscript $h O(n)$ indicates a homotopy orbit spectrum alias Borel construction. One can guess from Proposition 5.3, quoted earlier in this introduction, why $O(n)$ acts on the $n$-th derivative of $E$ at infinity. $O(n)$ also acts on $n V=\mathbb{R}^{n} \otimes V$ because it acts on $\mathbb{R}^{n}$. Finally $O(n)$ acts diagonally on the smash product. 
Example (to illustrate how this can be used). Let $E(V)=B \mathscr{G}(S(V))$. Then the map

$$
\eta_{1}: B \mathscr{G}=E\left(\mathbb{R}^{\infty}\right) \longrightarrow\left(T_{1} E\right)\left(\mathbb{R}^{\infty}\right)
$$

is not very useful-it is a homotopy equivalence. But the map of horizontal homotopy fibers in

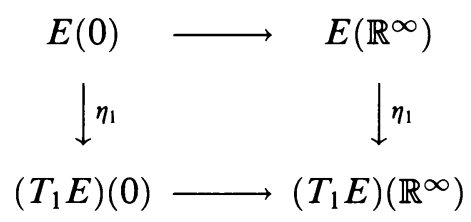

is useful, and by theorem 9.1 it takes the form

$$
\mathscr{G} \longrightarrow \Omega^{\infty}\left(\boldsymbol{\theta}_{h O(1)}\right) \text {. }
$$

Here $\boldsymbol{\theta}=\boldsymbol{\theta} E^{(1)}$ turns out to be a sphere spectrum, with the trivial action of $O(1)$. (Cf. Example 10.5.) So the codomain in (!) is the stable homotopy of projective space $\mathbb{R} P^{\infty}$. In fact, the map itself is "the" Kahn-Priddy map [KP].

Often theorem 9.1 is not "good enough" for applications, even when convergence takes place. It is easy to understand why by comparing Taylor towers (as above) to Postnikov towers. Let $X$ be a connected pointed $C W$-space, with Postnikov tower

$$
\cdots \rightarrow X_{[n+1]} \stackrel{p_{n+1}}{\longrightarrow} X_{[n]} \stackrel{p_{n}}{\longrightarrow} X_{[n-1]} \rightarrow \cdots
$$

(so that $X_{[i]}$ is the $i$-th Postnikov base of $X$, with vanishing $\pi_{j}$ for $j>i$ ). We can assume that the maps $p_{n}$ are fibrations. It is easy, especially nowadays, to say that the (homotopy) fiber of $p_{n}$ over the base point is an Eilenberg-Mac Lane space. It is more difficult to classify the fibration $p_{n}$, and this is especially true when $X$ is not simply connected.

Exactly the same problems arise in connection with the Taylor tower of a continuous functor $E$ from $\mathscr{J}$ to spaces. If we assume that $E\left(\mathbb{R}^{\infty}\right)$ is contractible-this corresponds to the assumption " $X$ is simply connected"then there is a simple solution.

Corollary 8.3, unravelled. For any $V$ in $\mathscr{J}$, any $n>0$, there is a fibration sequence up to homotopy

$$
\left(T_{n} E\right)(V) \stackrel{r_{n}}{\longrightarrow}\left(T_{n-1} E\right)(V) \longrightarrow \Omega^{\infty}\left[\left((n V)^{c} \wedge \Sigma \boldsymbol{\theta}\right)_{h O(n)}\right]
$$

where $\mathbf{\theta} \simeq \mathbf{8} E^{(n)}$ as in the theorem above. This is sufficiently natural in $V$.

When $E\left(\mathbb{R}^{\infty}\right)$ is not contractible, there is a more complicated solution. Suffice it to say that instead of a sequence of spectra

$$
\boldsymbol{\theta} E^{(1)}, \boldsymbol{\theta} E^{(2)}, \boldsymbol{8} E^{(3)}, \ldots
$$

the general solution (Corollary 8.5 and Rough Statement 8.8) involves a sequence of fibered spectra over the space $E\left(\mathbb{R}^{\infty}\right)$. From the analogy with Postnikov towers, this is exactly what one should expect.

Section 10 contains examples to illustrate the theory. Admittedly, they are hardly sufficient to justify the undertaking. More difficult and more interesting examples, at first derivative level, are to be found in [WW1] (continued in 
[WW2], [WW3] and [We]), and in [DWW]. At the level of second derivatives, there are fascinating questions, but few answers; I just hope that this will change.

I have made a point of stressing Goodwillie's influence on this paper. However, it has been pointed out to me that Goodwillie's calculus has an algebraic precursor in a paper by Eilenberg-Mac Lane [EMaL]; see esp. p. $92 \mathrm{ff}$. Also, the reader should realize that the category $\mathscr{J}$ and continuous functors from $\mathscr{J}$ to spaces (often with additional structure) are ubiquitous in the work of May, e.g. in [Ma].

Convention. The word space usually refers to a space which is compactly generated $[\mathrm{MaL}]$ and has the homotopy type of a $C W$-space. If it is pointed, then we assume that it is well-pointed (the inclusion of the base point has the homotopy extension property). In some real examples, however, a continuous functor $F$ from $\mathcal{J}$ to spaces may have some "bad" values. In the appendix, we discuss a method for improving such a functor.

\section{STIEFEL COMBinAtorics}

We will use capital letters $U, V, W$ to denote finite dimensional real vector spaces with inner product (positive definite). The one-point compactification of $V$ is $V^{c}$. For brevity, we write $n \cdot V$ to mean $\mathbb{R}^{n} \otimes V$. Let us think of $\mathbb{R}^{n}$ as a subspace of a suitable $\mathbb{R}^{\infty}$, so that $\mathbb{R}^{0} \subset \mathbb{R}^{1} \subset \mathbb{R}^{2} \ldots$. Then

$$
0 \cdot V \subset 1 \cdot V \subset 2 \cdot V \subset 3 \cdot V \ldots .
$$

Let $\operatorname{mor}(V, W)$ be the Stiefel manifold of linear maps from $V$ to $W$ preserving the inner product. We can make a category $\mathcal{J}$ with objects $U, V, W \ldots$ as above, and such that $\operatorname{mor}(V, W)$ is the set of morphisms from $V$ to $W$.

For each $n \geq 0$, let $\gamma_{n}(V, W)$ be the vector bundle on $\operatorname{mor}(V, W)$ whose total space consists of all pairs $(f, x)$ with $f$ in $\operatorname{mor}(V, W)$ and $x \in n$. coker $(f)$. (Think of coker $(f)$ as the orthogonal complement of $\operatorname{im}(f)$ in $W$.) Denote the Thom space of $\gamma_{n}(V, W)$ by $\operatorname{mor}_{n}(V, W)$. The composition law

$$
\operatorname{mor}(V, W) \times \operatorname{mor}(U, V) \longrightarrow \operatorname{mor}(U, W)
$$

is covered by a vector bundle map

$$
\gamma_{n}(V, W) \times \gamma_{n}(U, V) \longrightarrow \gamma_{n}(U, W)
$$

given by

$$
((f, x),(g, y)) \mapsto\left(f g, x+f_{*}(y)\right) .
$$

Passing to Thom spaces, we obtain a continuous composition law

$$
\operatorname{mor}_{n}(V, W) \wedge \operatorname{mor}_{n}(U, V) \longrightarrow \operatorname{mor}_{n}(U, W) .
$$

Let $\mathscr{J}_{n}$ be the category with objects $U, V, W, \ldots$ as above, and with $\operatorname{mor}_{n}(U, V)$ as the set of morphisms from $U$ to $V$. Composition of morphisms has been defined; we see that $\mathscr{J}_{n}$ is a pointed topological category with discrete class of objects. (The morphism sets are pointed topological spaces. The composition law for morphisms is continuous.) There are inclusions

$$
\mathscr{J}_{0} \subset \mathscr{J}_{1} \subset \mathscr{J}_{2} \subset \mathscr{J}_{3} \ldots .
$$

Note that $\mathscr{J}_{0}$ differs slightly from $\mathscr{J}$, in that $\operatorname{mor}_{0}(V, W)$ is $\operatorname{mor}(V, W)$ with an added base point. 
Later on we shall be interested in continuous functors $E$ from $\mathscr{J}_{0}$ to pointed spaces. Such a functor has a first derivative $E^{(1)}$, which is a continuous functor from $\mathscr{J}_{1}$ to pointed spaces. It also has a second derivative $E^{(2)}$, which is a continuous functor from $\mathscr{J}_{2}$ to pointed spaces, and so on. The idea is that $\mathscr{J}_{n}$ is the $n$-th jet category of $\mathscr{J}_{0}$.

Now we wish to understand the topology of the spaces $\operatorname{mor}_{n}(V, W)$ in some inductive sense (by induction on $n$, that is). Here is the simplest result in this direction.

\subsection{Proposition. The reduced mapping cone of the restriction map}

$$
\operatorname{mor}_{0}(\mathbb{R} \oplus V, W) \longrightarrow \operatorname{mor}_{0}(V, W)
$$

is homeomorphic to $\operatorname{mor}_{1}(V, W)$.

Proof. When $\operatorname{dim}(V) \geq \operatorname{dim}(W)$, the homeomorphism is obvious. We assume therefore that $\operatorname{dim}(V)<\operatorname{dim}(W)$; then the restriction map in 1.1 is onto, and its mapping cone is a quotient space of

$$
[0, \infty] \times \operatorname{mor}_{0}(\mathbb{R} \oplus V, W) .
$$

To an element in this quotient space, represented by $(t, f)$ say, we associate the element

$$
(f \mid V, t \cdot(f \mid \mathbb{R})(1)) \in \operatorname{mor}_{1}(V, W)
$$

provided $t \neq \infty$ and $f \neq *$. Otherwise $(t, f)$ represents the base point and must be mapped to the base point.

To find the correct generalization of 1.1 , we note that there is a "restricted" composition map

$$
\operatorname{mor}_{n}(\mathbb{R} \oplus V, W) \wedge\left(\mathbb{R}^{n}\right)^{c} \longrightarrow \operatorname{mor}_{n}(V, W) .
$$

(Here we identify $\left(\mathbb{R}^{n}\right)^{c}$ with the closure of the subspace of $\operatorname{mor}_{n}(V, \mathbb{R} \oplus V)$ consisting of all $(f, x)$ such that $f$ is the standard inclusion.) When $n=0$, this is just the restriction map of 1.1. Note also that the homeomorphism constructed in the proof of 1.1 has some naturality properties: it is a natural transformation between functors on $\mathscr{J}_{0}^{o p} \times \mathscr{J}_{0}$. (Since $\mathscr{J}_{0} \subset \mathscr{J}_{1}$, the rule taking a pair $(V, W)$ to $\operatorname{mor}_{1}(V, W)$ is a functor on $\mathscr{J}_{0}^{o p} \times \mathscr{J}_{0}$.)

1.2. Proposition. The reduced mapping cone of the restricted composition map

$$
\operatorname{mor}_{n}(\mathbb{R} \oplus V, W) \wedge\left(\mathbb{R}^{n}\right)^{c} \longrightarrow \operatorname{mor}_{n}(V, W)
$$

is homeomorphic to $\operatorname{mor}_{n+1}(V, W)$. The homeomorphism given below is a natural transformation between functors on $\mathscr{J}_{n}^{o p} \times \mathscr{J}_{n}$.

Proof. Again we can assume that $\operatorname{dim}(V)<\operatorname{dim}(W)$, and then the restricted composition map in 1.2. is onto (exercise). So the reduced mapping cone is a quotient space of

$$
[0, \infty] \times \operatorname{mor}_{n}(\mathbb{R} \oplus V) \times\left(\mathbb{R}^{n}\right)^{(c)} .
$$

Any element in this quotient space, distinct from the base point, is then represented by some $(t,(f, y), z)$ where

$$
\begin{aligned}
& t \in[0, \infty], \\
& f: \mathbb{R} \oplus V \rightarrow W, \\
& y \in n \cdot \operatorname{im}(f)^{\perp} \subset n \cdot W, \\
& z \in \mathbb{R}^{n} \cong n \cdot \mathbb{R} .
\end{aligned}
$$


To such a triple or quadruple we associate

$$
(g, x) \in \operatorname{mor}_{n+1}(V, W)
$$

where $g=f \mid V$, and

$$
x=y+(f \mid \mathbb{R})_{*}(z)+\alpha((f \mid \mathbb{R})(1)) .
$$

Here

$$
(f \mid \mathbb{R})_{*}: n \cdot \mathbb{R} \longrightarrow n \cdot W \subset(n+1) \cdot W
$$

is $n \cdot(f \mid \mathbb{R})$, and

$$
\alpha: W \hookrightarrow(n+1) \cdot W
$$

identifies $W$ with the (orthogonal) complement of $n \cdot W$ in $(n+1) \cdot W$.

Repeated application of Proposition 1.2 gives a description "up to extension problems" of $\operatorname{mor}_{n}(V, W)$ in terms of morphism spaces from $\mathscr{J}$. Let us see (in the next section) what this partial description is good for. We will find that it solves many problems, but not all.

\section{Derivatives}

Recall that a pointed space is well-pointed if the inclusion of the base point has the homotopy extension property. A functor $E$ from $\mathscr{J}_{n}$ to well-pointed spaces is pointed continuous if, for all $V, W$ in $\mathscr{J}$, the map

$$
\operatorname{mor}_{n}(V, W) \stackrel{E}{\longrightarrow} \operatorname{map}_{*}(E(V), E(W))
$$

is pointed and has a continuous adjoint

$$
\operatorname{mor}_{n}(V, W) \wedge E(V) \longrightarrow E(W) .
$$

Such functors $E$ and the natural transformations between them form a category which we denote by $\mathscr{E}_{n}$. A morphism $E \rightarrow F$ in $\mathscr{E}_{n}$ is an equivalence if $E(V) \rightarrow F(V)$ is a homotopy equivalence for all $V$. Two objects in $\mathscr{E}_{n}$ are equivalent if they can be related by a chain of equivalences.

We write nat $_{n}(E, F)$ for the set of natural transformations from $E$ to $F$ (in $\mathscr{E}_{n}$ ). For positive integers $m, n$ with $m \leq n$, restriction from $\mathscr{J}_{n}$ to $\mathscr{J}_{m}$ is a functor

$$
\operatorname{res}_{m}^{n}: \mathscr{E}_{n} \longrightarrow \mathscr{E}_{m}
$$

Remark. There is no obvious topology that we could put on the morphism sets nat $_{n}(E, F)$ in $\mathscr{Z}_{n}$. However, we can say that a map from a space $T$ to the set nat $_{n}(E, F)$ is continuous if its adjoint

$$
T_{+} \wedge E(V) \longrightarrow F(V)
$$

is continuous for all $V$. Briefly, $\operatorname{nat}_{n}(E, F)$ is a virtual space: a contravariant functor from spaces to sets having a suitable sheaf property. See [WW1, $\S 0$ ] for details. A natural transformation between two such functors will also be called a continuous map (if it is invertible, a homeomorphism). For example,

$$
\operatorname{res}_{m}^{n}: \operatorname{nat}_{n}(E, F) \longrightarrow \operatorname{nat}_{m}\left(\operatorname{res}_{m}^{n} E, \operatorname{res}_{m}^{n} F\right)
$$

is continuous. 
2.1. Proposition. The functor $\operatorname{res}_{m}^{n}$ has a right adjoint

$$
\operatorname{ind}_{m}^{n}: \mathscr{E}_{m} \longrightarrow \mathscr{E}_{n}
$$

Explanation. Adjointness means here that

$$
\operatorname{nat}_{n}\left(E, \operatorname{ind}_{m}^{n} F\right) \cong \operatorname{nat}_{m}\left(\operatorname{res}_{m}^{n} E, F\right)
$$

by a binatural bijection. The existence of $\operatorname{ind}_{m}^{n}$ is equivalent to the following statement (see [MaL,IV.7.Thm.3]): For each $F$ in $\mathscr{E}_{m}$, the comma category $\left(\operatorname{res}_{m}^{n} \downarrow F\right)$ has a terminal object. (The objects of the comma category are pairs $(E, g)$ where $E$ is an object of $\mathscr{E}_{n}$ and $g$ is a morphism in $\mathscr{E}_{m}$ from $\operatorname{res}_{m}^{n} E$ to $F$. A morphism from $\left(E_{1}, g_{1}\right)$ to $\left(E_{2}, g_{2}\right)$ is a morphism from $E_{1}$ to $E_{2}$ making the appropriate triangle commutative.) We can write the terminal object in the form $\left(\operatorname{ind}_{m}^{n} F, u_{F}\right)$, so that

$$
u_{F}: \operatorname{res}_{m}^{n} \operatorname{ind}_{m}^{n} F \longrightarrow F
$$

is "universal". The universal property characterizes $\operatorname{ind}_{m}^{n} F$ as the right Kan extension of $F$; see [MaL, X.3]. The word "extension" is a little misleading, though, since $\operatorname{ind}_{m}^{n} F$ hardly ever agrees with $F$ on $\mathscr{L}_{0}$.

Proof. We assume first that the right adjoint exists. Then, for $V$ in $\mathscr{J}_{n}$, we have

$$
\begin{aligned}
\operatorname{ind}_{m}^{n} F(V) & \cong \operatorname{nat}_{n}\left(\operatorname{mor}_{n}(V,-), \operatorname{ind}_{m}^{n} F\right) \quad \text { (by Yoneda's lemma) } \\
& \cong \operatorname{nat}_{m}\left(\operatorname{mor}_{n}(V,-), F\right) \quad \text { by adjointness. }
\end{aligned}
$$

We can use this last expression as a definition of the functor $\operatorname{ind}_{m}^{n} F$. As it stands, this is a functor from $\mathscr{J}_{n}$ to pointed virtual spaces. Define $u_{F}$ to be the restriction

$$
\operatorname{ind}_{m}^{n} F(V)=\operatorname{nat}_{m}\left(\operatorname{mor}_{n}(V,-), F\right) \longrightarrow \operatorname{nat}_{m}\left(\operatorname{mor}_{m}(V,-), F\right) \cong F(V)
$$

(for all $V$ ). To see that $u_{F}$ has the required universal property, let $(E, g)$ be an object of the comma category:

$$
g: \operatorname{res}_{m}^{n} E \longrightarrow F .
$$

Composition with $g$ is a continuous natural transformation

$$
E(V) \cong \operatorname{nat}_{n}\left(\operatorname{mor}_{n}(V,-), E\right) \longrightarrow \operatorname{nat}_{m}\left(\operatorname{mor}_{m}(V,-), F\right)=\operatorname{ind}_{m}^{n} F(V)
$$

making the appropriate triangle commutative. It is easily seen to be the only one.

To conclude the proof, we have to show that the virtual spaces $\operatorname{ind}_{m}^{n} F(V)$ are genuine spaces, for all $V$. It suffices to consider the case $n=m+1$. Namely, if ind $d_{m}^{m+1}$ exists, then ind ${ }_{m}^{n}$ exists and

$$
\operatorname{ind}_{m}^{n}=\operatorname{ind}_{n-1}^{n} \operatorname{ind}_{n-2}^{n-1} \ldots \operatorname{ind}_{m}^{m+1} .
$$

Now assume that $n=m+1$. We need a description of

$$
\operatorname{ind}_{m}^{m+1} F(V)=\operatorname{res}_{m}^{m+1} \operatorname{ind}_{m}^{m+1} F(V)=\operatorname{nat}_{m}\left(\operatorname{mor}_{m+1}(V,-), F\right),
$$

for all $V$. Since $F$ is a functor, we have evaluation maps

$$
\operatorname{mor}_{m}(V, \mathbb{R} \oplus V) \wedge F(V) \longrightarrow F(\mathbb{R} \oplus V) .
$$


We also have restricted evaluation maps

$$
\sigma:\left(\mathbb{R}^{m}\right)^{c} \wedge F(V) \longrightarrow F(\mathbb{R} \oplus V)
$$

because

$$
\left(\mathbb{R}^{m}\right)^{c} \subset \operatorname{mor}_{m}(V, \mathbb{R} \oplus V) .
$$

as the closure of the subspace consisting of all $(f, x)$ with $f$ equal to the standard inclusion. Here is the description we need.

2.2 Proposition. The functor $\operatorname{res}_{m}^{m+1} \operatorname{ind}_{m}^{m+1} F$ is isomorphic to the functor sending $V$ to the homotopy fiber of

$$
\sigma^{a d}: F(V) \longrightarrow \Omega^{m} F(\mathbb{R} \oplus V) .
$$

Under this isomorphism,

$$
u_{F}: \operatorname{res}_{m}^{m+1} \operatorname{ind}_{m}^{m+1} F \longrightarrow F
$$

corresponds to the forgetful map from the homotopy fiber to the domain of $\sigma^{\text {ad }}$. Proof. This follows directly from Proposition 1.2. (The proof of Proposition 2.1 is now complete.)

One useful consequence of Proposition 2.2 is the homotopy invariance of ind $_{m}^{m+1}$. In detail, suppose that $g$ is a morphism in $\mathscr{E}_{m}$ which is an equivalence (beginning of this section). Then ind $m_{m}^{m+1} g$ has the same property. This would not be obvious from Proposition 2.1 alone.

The proof of Proposition 2.2 suggests another description of $\mathscr{E}_{m}$. An object $E$ in $\mathscr{E}_{m}$ gives rise (by restriction) to a functor on $\mathscr{L}_{0}$, and to a natural transformation

$$
\sigma:(n V)^{c} \wedge E(W) \longrightarrow E(V \oplus W) .
$$

of functors on $\mathscr{J}_{0} \times \mathscr{J}_{0}$. (As usual, think of $(n V)^{c}$ as the closure of the subspace of $\operatorname{mor}_{m}(W, V \oplus W)$ consisting of all $(f, x)$ where $f$ is the standard inclusion. Define $\sigma$ by evaluation.) Since $E$ is a functor, $\sigma$ has an associativity property, and it agrees with the standard homeomorphism when $V=0$ and $W$ is arbitrary. It is not hard to recover $E$ from its restriction to $\mathscr{L}_{0}$ and the natural transformation $\sigma$.

From this point of view, it would be reasonable to say that an object in $\mathscr{E}_{m}$ for $m \geq 1$ is a coordinate free spectrum of multiplicity $m$. The case $m=1$ goes back to [Ma]. (May's definition is more rigid, though, and serves different purposes.) It also appears in [WW1, Definition 2.1], without the associativity condition on $\sigma$. At the time, we thought we had two good reasons for omitting the associativity condition: It appeared to be hard to arrange in practice, and it appeared to be automatically fulfilled in a homotopy theoretic sense, by [WW1, Proposition 2.2]. Both of these reasons have now disappeared; in particular, the proof of [WW1, Proposition 2.2] is incorrect.

We see that an object $E$ in $\mathscr{E}_{m}$ (where $m \geq 1$ ) gives rise to an "ordinary" spectrum $\boldsymbol{\theta} E$ made up from the spaces

$$
\Theta_{m k} E:=E\left(\mathbb{R}^{k}\right)
$$

The structure maps are specializations of $\sigma$, and take the form

$$
\Sigma^{m} \Theta_{m k} E \rightarrow \Theta_{m(k+1)} E .
$$


(It is quite enough to have $\Theta_{r} E$ defined for positive $r$ divisible by $m$ only.)

Strictly speaking, $\boldsymbol{\theta} E$ may not be a $C W$-spectrum in the sense of Boardman (see [Ad]), since the structure maps may not be inclusions, let alone cellular inclusions. Somewhat incorrectly, I shall call a map betwen "ordinary" spectra as above a homotopy equivalence if it induces an isomorphism on homotopy groups. For Boardman spectra, this is equivalent to the honest notion of homotopy equivalence.

2.3. Example. Write $\Omega^{V}$ for any space of maps from $V^{c}$ to something else (for $V$ in $\mathscr{J}$ ). Suppose that $E$ in $\mathscr{E}_{m}$ is such that

$$
\sigma^{a d}: E(W) \longrightarrow \Omega^{V} E(V \oplus W)
$$

is a homotopy equivalence for all $V, W$. (We may say that $E$ is stable.) If $m=$ 0 , this means that $E$ is essentially constant: There are natural transformations

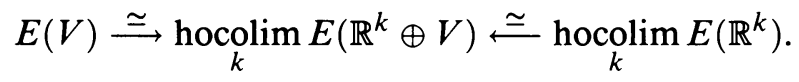

If $m \geq 0$, we have similarly

$$
E(V) \stackrel{\simeq}{\operatorname{hocolim}} \Omega^{m k} E\left(\mathbb{R}^{k} \oplus V\right) \stackrel{\simeq}{\underset{k}{\operatorname{hocolim}} \Omega^{m k}}\left((m V)^{c} \wedge E\left(\mathbb{R}^{k}\right)\right)
$$

where the map on the left is an inclusion, the homotopy direct limits are defined using $\sigma$, and the map on the right is again made from $\sigma$. Note that the domain of this last map is just

$$
\Omega^{\infty}\left((m V)^{c} \wedge \boldsymbol{\theta} E\right) .
$$

Conversely, if $\boldsymbol{\theta}$ is any spectrum (made from well-pointed spaces), then the functor $E$ given by

$$
V \longmapsto \Omega^{\infty}\left((m V)^{c} \wedge \boldsymbol{\theta}\right)
$$

on $\mathscr{J}_{0}$ comes with an evident natural transformation

$$
\sigma:(m V)^{c} \wedge E(W) \longrightarrow E(V \oplus W) .
$$

Hence a stable object in $\mathscr{E}_{m}$ is determined (up to equivalence) by its spectrum $\boldsymbol{\theta} E$, which can be chosen arbitrarily. In this sense, stable objects are "constant". If $E$ in $\mathscr{E}_{m}$ is stable, then $\operatorname{ind}_{m}^{m+1} E$ vanishes (has contractible values) by Proposition 2.2. The converse is not quite true; we will return to this point in section 5. Still, it seems reasonable to think of $\operatorname{ind}_{m}^{m+1} E$ as the rate of change of $E$.

2.5. Notation. We abbreviate

$$
E^{(n-m)}:=\operatorname{ind}_{m}^{n} E \quad \text { for } n \geq m
$$

We call $E^{(1)}$ the derivative of $E$.

2.6. Remark. (This is sometimes useful in identifying derivatives.) Suppose that

$$
g: \operatorname{res}_{m}^{m+1} F \longrightarrow E
$$

is a morphism in $\mathscr{E}_{m}$ (with $F$ in $\mathscr{E}_{m+1}$, and $E$ in $\mathscr{E}_{m}$ ). The adjoint of $g$ is a natural transformation

$$
F(V) \longrightarrow E^{(1)}(V) \cong \text { hofiber }[E(V) \rightarrow E(\mathbb{R} \oplus V)]
$$


It refines $g$ and has the following explicit description. For each $V$, the composition

$$
F(V) \stackrel{g}{\longrightarrow} E(V) \stackrel{\sigma_{E}^{a d}}{\longrightarrow} \Omega^{m} E(\mathbb{R} \oplus V)
$$

is nullhomotopic because it equals the composition

$$
F(V) \stackrel{\sigma_{F}^{a d}}{\longrightarrow} \Omega^{m+1} F(\mathbb{R} \oplus V) \stackrel{r}{\longrightarrow} \Omega^{m} F(\mathbb{R} \oplus V) \stackrel{g}{\longrightarrow} \Omega^{m} E(\mathbb{R} \oplus V)
$$

where $r$ is restriction. (Clearly $r$ is canonically nullhomotopic.)

Usually we start with an object $F$ in $\mathscr{E}_{0}$, form the derivatives $F^{(1)}, F^{(2)}$, $F^{(3)}, \ldots$, and then (for reasons explained in the introduction) try to "calculate" the ordinary spectra $\boldsymbol{\Theta} F^{(1)}, \boldsymbol{\Theta} F^{(2)}, \boldsymbol{\Theta} F^{(3)}, \ldots$ to some extent. Here comes the standard example.

2.7. Example. Take $F(V)=B O(V)$ as in the introduction. Then

$$
F^{(1)}(V) \simeq O(\mathbb{R} \oplus V) / O(V) \cong V^{c}
$$

by Proposition 2.2. Hence a good candidate for $F^{(1)}$ is $E$ in $\mathscr{E}_{1}$ given by $E(V)=V^{c}$ and by

$$
E(f, x)(v):=f(v)+x \quad \text { for }(f, x) \in \operatorname{mor}_{1}(V, W) \text { and } v \in V .
$$

We already have an interesting morphism

$$
g: \operatorname{res}_{0}^{1} E \longrightarrow F
$$

(for each $V$, the usual map from $O(\mathbb{R} \oplus V) / O(V)$ to $B O(V)$ ). Its adjoint is a natural homotopy equivalence

$$
E \longrightarrow F^{(1)}
$$

by Remark 2.6. So our guess was correct, and $\boldsymbol{\Theta} F^{(1)}$ is a sphere spectrum,

$$
\mathbf{\theta} F^{(1)} \simeq \mathbf{S}^{0} .
$$

Next, $\boldsymbol{\Theta} F^{(2)}$ is homotopy equivalent to $\boldsymbol{\Theta} E^{(1)}$, and this in turn is homotopy equivalent to $\Omega^{2} \mathbf{\Theta} D^{(1)}$ where

$$
D(V)=E(\mathbb{R} \oplus V)=\Sigma V^{c} .
$$

By Proposition 2.2, we have

$$
\Theta_{2 k} D^{(1)}=D^{(1)}\left(\mathbb{R}^{k}\right)=\text { hofiber }\left[\sigma^{a d}: S^{k+1} \rightarrow \Omega \Sigma S^{k+1}\right],
$$

and the James model [Ja] for $\Omega \Sigma S^{k+1}$ suggests immediately that $\boldsymbol{\Theta} D^{(1)}$ is homotopy equivalent to a shifted sphere spectrum $\mathbf{S}^{1}$. To prove this, we can use Remark 2.6 once more. Accordingly, we attempt to construct an "easy" object $C$ in $\mathscr{E}_{2}$ and a morphism

$$
h: \operatorname{res}_{1}^{2} C \longrightarrow D
$$

in $\mathscr{E}_{1}$ whose adjoint $C \longrightarrow D^{(1)}$ induces a homotopy equivalence $\boldsymbol{\theta} C \simeq \mathbf{\theta} D^{(1)}$ for some "easy" reason. The James model for $\Omega \Sigma D(V)$ suggests that

$$
C(V):=\Sigma\left(V^{c} \wedge V^{c}\right)
$$

is a good definition, and that $h: C(V) \longrightarrow D(V)$ should be the Whitehead bracket

$$
[l, l]: \Sigma\left(V^{c} \wedge V^{c}\right) \longrightarrow \Sigma V^{c} .
$$


This is easily seen to work, and we conclude that the spectra $\boldsymbol{\theta} F^{(2)}, \boldsymbol{\theta} E^{(1)}$, $\Omega^{2} \boldsymbol{\theta} D^{(1)}$ are homotopy equivalent to $\Omega^{2} \boldsymbol{\theta} C$. This means that

$$
\boldsymbol{\theta} F^{(2)} \simeq \mathbf{S}^{-1} \text {. }
$$

In order to determine $\boldsymbol{\theta}_{F}^{(3)}$, we need a rudimentary knowledge of $F^{(3)}$, and therefore a more thorough understanding of $F^{(2)}$, or equivalently, of $E^{(1)}$. Since $E^{(1)}(V)$ is the homotopy fiber of a map between spaces with manageable cohomology, we can use the Eilenberg-Moore spectral sequence [EM] to determine its cohomology. The James model for $\Omega \Sigma V^{c}$ (which also models the multiplication) shows that the Pontryagin algebra $H_{*}\left(\Omega \Sigma V^{c}\right)$ is a polynomial algebra $\mathbb{Z}[x]$ where $|x|=n=\operatorname{dim}(V)$. Write $x^{0}, x^{1}, x^{2}, x^{3}, \ldots$ for the powers of $x$, and write $1, x_{1}, x_{2}, x_{3}$ for their duals in $H^{*}\left(\Omega \Sigma V^{c}\right)$. The cup multiplication in $H^{*}\left(\Omega \Sigma V^{c}\right)$ is given by

$$
\left(x_{1}\right)^{k}=k ! x_{k} \quad \text { if } n \text { is even, }
$$

and by

$$
\left(x_{1}\right)^{2}=0, \quad x_{1} x_{2 k}=x_{2 k+1}, \quad\left(x_{2}\right)^{k}=k ! x_{2 k} \quad \text { if } n \text { is odd. }
$$

(The comultiplication leaves no other choice.) The $E_{2}^{* *}$ term of the appropriate Eilenberg-Moore spectral sequence is

$$
\operatorname{Tor}_{R}^{* *}\left(H^{*}\left(V^{c}\right), \mathbb{Z}\right),
$$

where $R=H^{*}\left(\Omega \Sigma V^{c}\right)$. The spectral sequence converges to the cohomology of $F^{(2)}(V)$. It collapses for trivial reasons in the relevant range, showing for even $n=\operatorname{dim}(V)$ that

$$
\begin{aligned}
H^{0}\left(F^{(2)}(V)\right) & \cong H^{2 n-1}\left(F^{(2)}(V)\right) \cong \mathbb{Z}, \\
H^{3 n-1}\left(F^{(2)}(V)\right) & \cong \mathbb{Z} / 3, \\
H^{k}\left(F^{(2)}(V)\right) & =0 \quad \text { for all other } k<4 n-2, \\
H^{k}\left(F^{(2)}(V) ; \mathbb{Q}\right) & =0 \quad \text { for all } k \geq 4 n-2,
\end{aligned}
$$

and for odd $n$ that

$$
\begin{aligned}
H^{0}\left(F^{(2)}(V)\right) & \cong H^{2 n-1}\left(F^{(2)}(V)\right) \cong \mathbb{Z}, \\
H^{k}\left(F^{(2)}(V)\right) & =0 \quad \text { for all other } k<4 n-2, \\
H^{k}\left(F^{(2)}(V) ; \mathbb{Q}\right) & =0 \quad \text { for all } k \geq 4 n-2 .
\end{aligned}
$$

This implies, together with what we already know about $F^{(2)}$ as a functor, that

$$
\begin{aligned}
H_{3 n-2}\left(F^{(3)}(V)\right) & \cong \mathbb{Z} / 3 & & \\
H_{k}\left(F^{(3)}(V)\right) & =0 & & \text { for } 0<k<4 n-2 \text { and } k \neq 3 n-2, \\
H_{k}\left(F^{(3)}(V) ; \mathbb{Q}\right) & =0 & & \text { for all } k>0 .
\end{aligned}
$$

It is very difficult to figure out what the suspension maps

$$
\sigma^{(3)}: \Sigma^{3} F^{(3)}(V) \longrightarrow F^{(3)}(\mathbb{R} \oplus V)
$$

do on homology $H_{3 n+1}$. Leaving this for the moment, we can only say that $\boldsymbol{\theta} F^{(3)}$ is either a shifted $\mathbb{Z} / 3$-Moore spectrum,

$$
\boldsymbol{\theta} F^{(3)} \simeq \Omega^{2} \operatorname{mo}(3),
$$


or that $\mathbf{\theta} F^{(3)}$ is contractible. We shall settle the issue later-it turns out that the first guess is correct. The calculation above was shown to me by Tom Goodwillie in 1988, and again by John Klein in 1991. (They used somewhat different methods.)

Now it seems very hard to guess what $\mathbf{\Theta} F^{(4)}$ might be. Fortunately, Tom Goodwillie has found something like a closed formula for all the $\mathbf{\Theta} F^{(n)}$, using a relationship between his own calculus and the orthogonal calculus.

\section{SYMMETRY}

For each $n \geq 0$, the pointed topological category $\mathscr{I}_{n}$ comes with a remarkable left action of the orthogonal group $O(n)$. The action leaves objects fixed and is given on nonzero morphisms by

$$
t(f, x):=(f, t x) \quad \text { for } t \in O(n) .
$$

(Here $x$ belongs to $n \cdot \operatorname{coker}(f)=\mathbb{R}^{n} \otimes \operatorname{coker}(f)$, where $O(n)$ acts because it acts on $\mathbb{R}^{n}$.) Note that the action is trivial on $\mathscr{J}_{0} \subset \mathscr{J}_{n}$. We write $\lambda(t)$ for the automorphism of $\mathscr{J}_{n}$ corresponding to $t \in O(n)$.

Next, $O(n)$ acts on the left of $\mathscr{E}_{n}$. It acts on objects $E$ by

$$
t E:=E \lambda\left(t^{-1}\right),
$$

and on morphisms $g: E \rightarrow F$ by

$$
\operatorname{tg}:=g \text {. }
$$

(The second formula is meaningful because $t E(V)=E(V)$ and $t F(V)=$ $F(V)$.) We note that

$$
\operatorname{res}_{0}^{n}(t E)=\operatorname{res}_{0}^{n} E, \quad \operatorname{res}_{0}^{n}(t g)=\operatorname{res}_{0}^{n}(g),
$$

which means that the action is over $\mathscr{E}_{0}$.

In section 2 we constructed among other things a right adjoint

$$
F \longmapsto F^{(n)}=\operatorname{ind}_{0}^{n} F
$$

for the restriction functor $\operatorname{res}_{0}^{n}$. Now the functor

$$
F \longmapsto t\left(F^{(n)}\right)
$$

from $\mathscr{E}_{0}$ to $\mathscr{E}_{n}$ is clearly also right adjoint to $\operatorname{res}_{0}^{n}$, for fixed $t \in O(n)$. By the uniqueness of right adjoints, or more directly by the uniqueness of universal objects, there exists a unique isomorphism

$$
a_{t}: F^{(n)} \longrightarrow t\left(F^{(n)}\right)
$$

in $\mathscr{E}_{n}$ such that (in shorthand notation)

$$
u_{F} a_{t}=u_{F}
$$

where $u_{F}$ is the universal morphism from $\operatorname{res}_{0}^{n} F^{(n)}$ to $F$. (See the explanation following Proposition 2.1.) Noting once more that $F^{(n)}$ agrees with $t\left(F^{(n)}\right)$ on objects, we have a result about the symmetry properties of $F^{(n)}$. 
3.1. Proposition. There exists a unique family $\left\{\alpha_{V}\right\}$ of left actions

$$
\alpha_{V}: O(n) \times F^{(n)}(V) \longrightarrow F^{(n)}(V)
$$

making the maps

$$
\begin{gathered}
\text { evaluation }: \operatorname{mor}_{n}(V, W) \wedge F^{(n)}(V) \longrightarrow F^{(n)}(W) \\
u: F^{(n)}(V) \longrightarrow F(V)
\end{gathered}
$$

into $O(n)$-maps. (Here $O(n)$ acts diagonally on the domain of the evaluation map, and trivially on $F(V)$.)

For an explicit description of $\alpha_{V}$, use the definition

$$
F^{(n)}(V)=\operatorname{nat}_{0}\left(\operatorname{mor}_{n}(V,-), F\right)
$$

from the proof of Proposition 2.1 and let $\alpha_{V}(t, g)=g \lambda\left(t^{-1}\right)$ for $g \in F^{(n)}(V)$ and $t \in O(n)$. The explicit description shows that $\alpha_{V}$ is continuous (as a map of virtual spaces, therefore as a map of spaces).

3.2. Example. For a smooth compact manifold $M$, let

$$
F(V)=B \operatorname{DIFF}^{b}(M \times V),
$$

where $\operatorname{DIFF}^{b}(M \times V)$ is the group of bounded diffeomorphisms from $M \times V$ to $M \times V$ which are the identity on $\partial M \times V$. (A continuous $f=\left(f_{1}, f_{2}\right)$ from $M \times V$ to $M \times V$ is bounded if there exists $c>0$ such that the norm of $f_{2}(m, v)-v$ is less than $c$, for all $(m, v) \in M \times V$. See [WW1] for more details, and see the appendix for questions regarding the topology on $\operatorname{DIFF}^{b}(M \times V)$.)

Then $\Omega F^{(1)}(V)$ is homotopy equivalent to the homotopy fiber of the inclusion map

$$
\operatorname{DIFF}^{b}(M \times V) \hookrightarrow \operatorname{DIFF}^{b}(M \times(V \oplus \mathbb{R}))
$$

which is homotopy equivalent to the space $\mathscr{C}^{b}(M \times V)$ of smooth bounded concordances. See [WW1, Proposition 1.8] for a proof of this statement in the TOP setting (it goes back to $[\mathrm{AnH}]$ and [Ha]). Taking $V=\mathbb{R}^{n}$ we have

$$
\Omega^{n} \mathscr{C}^{b}\left(M \times \mathbb{R}^{n}\right) \simeq \mathscr{C}^{b}\left(M \times D^{n}\right),
$$

again by [AnH] or [WW1, Proposition 1.5]. The arguments of [WW1, Definition 1.11, Proposition 1.12] show that $\boldsymbol{\theta} F^{(1)}$ is $\Omega \mathrm{Wh}^{\mathrm{DIFF}}(M)$, the smooth (usually nonconnective) Whitehead spectrum of $M$.

With the above identifications,

$$
\Omega^{n+1} \sigma^{a d}: \Omega^{n+1} F^{(1)}\left(\mathbb{R}^{n}\right) \longrightarrow \Omega^{n+2} F^{(1)}\left(\mathbb{R}^{n+1}\right)
$$

becomes the usual stabilization map

$$
\mathscr{C}^{b}\left(M \times D^{n}\right) \longrightarrow \mathscr{C}^{b}\left(M \times D^{n+1}\right)
$$

and $\Omega^{n+1} F^{(2)}\left(\mathbb{R}^{n}\right)$ becomes the homotopy fiber $\phi_{n}(M)$ of the usual stabilization map. Now Proposition 3.1 tells us that $\phi_{n}(M)$ comes with a canonical $O(2)$-action-a not so well known fact, although the action can be described by elementary geometric means. Further, Propositions 2.1 and 2.2 tell us among other things that there are stabilization maps

$$
\phi_{n}(M) \longrightarrow \Omega \phi_{n+1}(M)
$$

(namely, the maps $\Omega^{n+1} \sigma_{a d}$ ). This is not too well known either. The spectrum made from these spaces $\phi_{n}(M)$ and from these suspension maps is, of course, homotopy equivalent to $\Omega \mathbf{\Theta} F^{(2)}$. 
3.3. Definition. A symmetric object in $\mathscr{E}_{n}$ is an object $E$ together with continuous actions

$$
O(n) \times E(V) \longrightarrow E(V)
$$

(one for each $V$ ) such that the evaluation maps

$$
\operatorname{mor}_{n}(V, W) \wedge E(V) \longrightarrow E(W)
$$

are $O(n)$-maps. (Use the diagonal action on the domain of the evaluation map.)

By Proposition 3.1, the functor $F^{(n)}$ is symmetric for any $F$ in $\mathscr{E}_{0}$. In general, if $E$ in $\mathscr{E}_{n}$ is symmetric, then the maps

$$
\sigma:\left(\mathbb{R}^{n}\right)^{c} \wedge E\left(\mathbb{R}^{k}\right) \longrightarrow E\left(\mathbb{R}^{k+1}\right)
$$

defining the spectrum $\boldsymbol{\theta} E$ are $O(n)$-maps for the diagonal action on the domain. This suggests that $O(n)$ acts "somehow" on $\boldsymbol{\theta E}$. But it is not quite clear how, since an honest action should leave the suspension coordinates alone. Here we can apply a trick from equivariant homotopy theory.

Suppose that $\boldsymbol{\theta}$ is any spectrum, made from spaces $\boldsymbol{\theta}_{k}$ and maps $\Sigma \Theta_{k} \rightarrow$ $\Theta_{k+1}$. Then the spaces and maps

$$
\begin{gathered}
\Theta_{k}^{\sharp}:=\Omega^{\infty}\left(S^{k} \wedge \boldsymbol{\theta}\right), \\
\Sigma \Omega^{\infty}\left(S^{k} \wedge \boldsymbol{\theta}\right) \hookrightarrow \Omega^{\infty}\left(\Sigma S^{k} \wedge \boldsymbol{\theta}\right) \cong \Omega^{\infty}\left(S^{k+1} \wedge \boldsymbol{\theta}\right)
\end{gathered}
$$

constitute a new spectrum $\boldsymbol{\Theta}^{\sharp}$. This contains a copy of $\boldsymbol{\theta}$, since

$$
\boldsymbol{\Theta}_{k} \hookrightarrow \Omega^{k}\left(S^{k} \wedge \Theta_{k}\right) \hookrightarrow \Theta_{k}^{\sharp} .
$$

The inclusion $\boldsymbol{\theta} \hookrightarrow \boldsymbol{\theta}^{\sharp}$ is a homotopy equivalence.

Of course, all this works just as well if $\boldsymbol{\Theta}_{k}$ is defined for positive $k$ divisible by $n$ only (in which case $\Theta_{k}^{\sharp}$ is nevertheless defined for all positive $k$ ). Hence we can apply it with $\boldsymbol{\theta}=\boldsymbol{\theta} E$. Then each $\Theta_{k}^{\sharp}$, and more generally each

$$
\Omega^{\infty}(X \wedge \boldsymbol{\theta})=\underset{j}{\operatorname{hocolim}} \Omega^{j n}\left(X \wedge E\left(\mathbb{R}^{j}\right)\right)
$$

(for a $C W$-space $X$ ) comes with an action of $O(n)$ given by

$$
(t \cdot f)(v):=t\left(f\left(t^{-1}\right)\right)
$$

for $t \in O(n)$ and $v \in j \cdot \mathbb{R}^{n}$ and

$$
f:\left(j \cdot \mathbb{R}^{n}\right)^{c} \rightarrow X \wedge E\left(\mathbb{R}^{j}\right) .
$$

(Use the standard action of $O(n)$ on $\mathbb{R}^{n}$, and the action on $E\left(\mathbb{R}^{j}\right)$ coming from Definition 3.3.) The action is natural in $X$, and it follows that the maps

$$
\Sigma \Theta_{k}^{\sharp} \longrightarrow \Theta_{k+1}^{\sharp}
$$

defined above are $O(n)$-maps, with the suspended action on the domain. To summarize, $O(n)$ acts on $\boldsymbol{\theta}^{\sharp}=\boldsymbol{\theta}^{\sharp} E$ which is homotopy equivalent to $\boldsymbol{\theta} E$.

\section{More Stiefel combinatorics}

Which objects $E$ in $\mathscr{E}_{0}$ should be called polynomial functors of degree $\leq$ $n$ ? If we want to do "calculus", then we should certainly answer this question at some point. One obvious requirement is that the $(n+1)$-th derivative of 
$E$ should vanish (i.e., that $E^{(n+1)}(V)$ be contractible for all $V$ ). However, the case $n=0$ shows that this is not enough. Clearly $E$ should be called polynomial of degree 0 if and only if $E(f)$ is a homotopy equivalence for all nonzero morphisms $f$ in $\mathscr{J}_{0}$. According to this belief, the functor $E$ given by

$$
\begin{aligned}
& E(V)=S^{0} \quad \text { when } \operatorname{dim}(V)>5, \\
& E(V)=* \quad \text { when } \operatorname{dim}(V) \leq 5, \\
& E(f)=\text { identity whenever possible }
\end{aligned}
$$

is not polynomial of degree 0 . But $E^{(1)}$ does vanish.

Here it is clear what is happening: $E^{(1)}(V)$ is the homotopy fiber of a map from $E(V)$ to $E(\mathbb{R} \oplus V)$. If the map is a homotopy equivalence, for all $V$, then $E$ is polynomial of degree 0 . In that case $E^{(1)}$ vanishes, but the converse is false.

For arbitrary $n \geq 0$, we may hope that $E^{(n+1)}(V)$ can be identified with the homotopy fiber of a natural map

$$
g_{V}: E(V) \longrightarrow ?(V)
$$

where ? is a new object in $\mathscr{E}_{0}$, depending on $E$, that we have to create. We could then say that $E$ is polynomial of degree $\leq n$ if $g_{V}$ is a homotopy equivalence for all $V$. All this shows that a better understanding of $E^{(n+1)}$ is necessary. What we know about $E^{(n+1)}$ comes from section 1 (via Proposition 2.2). It now appears that we did not go far enough in section 1. In fact, Proposition 1.2 suggests (by downward induction on $n$ ) that there exists a description of $\operatorname{mor}_{n+1}(V, W)$ in terms of morphism sets in $\mathscr{J}_{0}$. Such a description does exist. It involves homotopy colimits. Let us recall the definition [BK].

A functor $Z$ from a small category $\mathscr{C}$ to spaces gives rise to a simplicial space

$$
[n] \quad \longmapsto \quad \coprod_{G:[n] \rightarrow \mathscr{C}} Z G(0)
$$

(Here $[n]$ is the ordered set $\{0,1, \ldots, n\}$, and $G$ denotes functors from $[n]$ to $\mathscr{C}$.) The homotopy colimit of $Z$ is the geometric realization of this simplicial space. Dually, the homotopy limit of $Z$ is the totalization [BK,Ch.X] of the cosimplicial space

$$
[n] \quad \longmapsto \quad \prod_{G:[n] \rightarrow \mathscr{C}} Z G(n) .
$$

(The totalization of a cosimplicial space $[n] \mapsto \mathfrak{Y}[n]$ is the space of natural transformations from $[n] \mapsto \triangle^{n}$ to $\left.[n] \mapsto \mathfrak{Y}[n].\right)$

In many examples, $\mathscr{C}$ is a topological category and $Z$ is a continuous functor. When this is the case, the homotopy (co-)limit should be modified. Instead of going into details, let us just consider the example that we shall need in Theorem 4.1 below.

Let $\mathscr{C}$ be the poset of all nonzero linear subspaces of $\mathbb{R}^{n+1}$, ordered by reversed inclusion: $T \leq U$ means $U \subset T$. Then $\mathrm{Ob}(\mathscr{C})$ is topologized as a disjoint union of Grassmannians. The set of functors

$$
G:[n] \rightarrow \mathscr{C}
$$


is also topologized for any $n \geq 0$, as a disjoint union of flag manifolds. Fix objects $V$ and $W$ in $\mathscr{L}_{0}$ and let

$$
Z(U):=\operatorname{mor}(U \oplus V, W) .
$$

(This is covariant.) Then the (set-theoretic) coproduct

$$
\coprod_{G:[n] \rightarrow \mathscr{C}} Z G(0)
$$

is again topologized, in such a way that the projection to the space

$$
\{G:[n] \rightarrow \mathscr{C}\}
$$

is a smooth manifold fiber bundle. We use these topologies to make a simplicial space

$$
[n] \quad \longmapsto \coprod_{G:[n] \rightarrow \mathscr{C}} Z G(0),
$$

and call its geometric realization the (topological) homotopy colimit of $Z$. Restriction gives a natural transformation from $Z$ to the constant functor with value $\operatorname{mor}(V, W)$. This induces a restriction map from hocolim $Z$ to $\operatorname{mor}(V, W)$. Now, in order to be informal and informative, we just write

$$
\text { hocolim } Z=\underset{0 \neq U \subset \mathbb{R}^{n+1}}{\operatorname{hocolim}} \operatorname{mor}(U \oplus V, W) \text {, }
$$

and we are ready for the theorem.

4.1. Theorem. The (unreduced) mapping cone of the restriction map

$$
\underset{\substack{\operatorname{hocolim} \\ 0 \neq U \subset \mathbb{R}^{n+1}}}{\operatorname{mor}}(U \oplus V, W) \longrightarrow \operatorname{mor}(V, W)
$$

is homeomorphic to $\operatorname{mor}_{n+1}(V, W)$. The homeomorphism specified below is pointed, and relative to $\operatorname{mor}(V, W)$, and it is natural in $V$ and $W$. More precisely: it is a natural transformation between functors on $\mathscr{J}^{o p} \times \mathscr{J}$.

Proof. We shall construct a homeomorphism betwen the respective complements of $\operatorname{mor}(V, W)$, and extend it (continuously) using the identity on $\operatorname{mor}(V, W)$. Note that the complement of $\operatorname{mor}(V, W)_{+}$in the big mapping cone has the form

$$
] 0, \infty[\times \underset{U}{\operatorname{hocolim}} \operatorname{mor}(U \oplus V, W)
$$

(where mor is for Stiefel manifolds). Let

$$
(f, x) \in \operatorname{mor}_{n+1}(V, W) \backslash \operatorname{mor}(V, W)_{+},
$$

so that $f$ from $V$ to $W$ is linear, preserves the inner product, and

$$
0 \neq x \in(n+1) \cdot \operatorname{coker}(f) \cong \operatorname{hom}\left(\mathbb{R}^{n+1}, \operatorname{coker}(f)\right) .
$$

Here we identify $\operatorname{coker}(f)$ with the orthogonal complement of $\operatorname{im}(f)$ in $W$. Since both $\mathbb{R}^{n+1}$ and coker $(f)$ are now equipped with inner products, the linear map

has an adjoint

$$
x: \mathbb{R}^{n+1} \longrightarrow \operatorname{coker}(f)
$$

$$
x^{*}: \operatorname{coker}(f) \longrightarrow \mathbb{R}^{n+1} \text {. }
$$


Then $x^{*} x: \mathbb{R}^{n+1} \rightarrow \mathbb{R}^{n+1}$ is self-adjoint, alias symmetric. We split $\mathbb{R}^{n+1}$ into an orthogonal sum of eigenspaces of $x^{*} x$,

$$
\mathbb{R}^{n+1} \cong \operatorname{ker}\left(x^{*} x\right) \oplus E\left(\lambda_{0}\right) \oplus E\left(\lambda_{1}\right) \oplus \cdots \oplus E\left(\lambda_{k}\right)
$$

where $\lambda_{0}<\lambda_{1}<\cdots<\lambda_{k}$ are the nonzero eigenvalues. (They are real and positive. Note that $k>0$ since $x \neq 0$.) Given all this, we make it into

(1) a functor $G:[k] \rightarrow \mathscr{C}$ (where $\mathscr{C}$ is still the poset of nonzero linear subspaces of $\mathbb{R}^{n+1}$, ordered by reversed inclusion);

(2) a point $z$ in $\operatorname{mor}(G(0) \oplus V, W)$;

(3) a point $p$ in the simplex $\Delta^{k}$;

(4) a real number $t>0$.

The functor $G$ is given by

$$
r \longmapsto E\left(\lambda_{0}\right) \oplus E\left(\lambda_{1}\right) \oplus \cdots \oplus E\left(\lambda_{k-r}\right) .
$$

The morphism $z$ is given by $z=f$ on $V$, and $z=\left(\lambda_{i}\right)^{-1 / 2} \cdot f$ on $E\left(\lambda_{i}\right)$. The point $p$ is, in barycentric coordinates,

$$
\lambda_{k}^{-1} \cdot\left(\lambda_{0}, \lambda_{1}-\lambda_{0}, \ldots, \lambda_{k}-\lambda_{k-1}\right) \text {. }
$$

The real number $t$ is just $\lambda_{k}$.

Finally we note that $G, z$ and $p$ together define a point in

$$
\underset{0 \neq U \subset \mathbb{R}^{n+1}}{\operatorname{hocolim}} \operatorname{mor}(U \oplus V, W)
$$

because the hocolim is defined to be a certain quotient of

$$
\coprod_{k \geq 0} \coprod_{G:[k] \rightarrow \mathscr{C}} \operatorname{mor}(G(0) \oplus V, W) \times \triangle_{+}^{k}
$$

(as a set). Starting from $(f, x)$ as above, we have therefore constructed a point in

$$
] 0, \infty[\times \underset{U}{\operatorname{hocolim}} \operatorname{mor}(U \oplus V, W)
$$

with first coordinate $t$. This is what we wanted. The construction is easily seen to be a homeomorphism, and it extends to the promised relative homeomorphism.

Recall from section 1 that $\operatorname{mor}_{n+1}(V, W)$ is the Thom space of a Riemannian vector bundle $\gamma_{n+1}(V, W)$ on the Stiefel manifold $\operatorname{mor}(V, W)$. Let $S \gamma_{n+1}(V, W)$ be the total space of the corresponding unit sphere bundle.

\subsection{Proposition. There is a natural homeomorphism}

$$
\underset{0 \neq U \subset \mathbb{R}^{n+1}}{\operatorname{hocolim}} \operatorname{mor}(U \oplus V, W) \longrightarrow S \gamma_{n+1}(V, W)
$$

for $V, W$ in $\mathscr{J}$.

Proof. This is already contained in the proof of Proposition 4.1, where we constructed a homeomorphism

$$
] 0, \infty\left[\times \underset{U}{\operatorname{hocolim}} \operatorname{mor}(U \oplus V, W) \longrightarrow \operatorname{mor}_{n+1}(V, W) \backslash \operatorname{mor}(V, W)_{+} \cdot\right.
$$

Compose with the inclusion of $\{1\} \times$ hocolim ..., and project

$$
\left.\operatorname{mor}_{n+1}(V, W) \backslash \operatorname{mor}(V, W)_{+} \cong\right] 0, \infty\left[\times S \gamma_{n+1}(V, W)\right.
$$

to $S \gamma_{n+1}(V, W)$. 
The next proposition is a useful supplement to Proposition 4.2. We write $\varepsilon^{n}$ for a trivial $n$-dimensional vector bundle.

\subsection{Proposition. For the restriction map}

$$
\text { res : } \operatorname{mor}(\mathbb{R} \oplus V, W) \longrightarrow \operatorname{mor}(V, W),
$$

we have

$$
\operatorname{res}^{*} \gamma_{n}(V, W) \cong \varepsilon^{n} \oplus \gamma_{n}(\mathbb{R} \oplus V, W) .
$$

Proof. The general case follows from the case $n=1$ by taking tensor product with $\epsilon^{n}$. Assume $n=1$, and fix a point $f$ in the base space $\operatorname{mor}(\mathbb{R} \oplus V, W)$. The two fibers (vector spaces) to be compared are then $\operatorname{coker}(f \mid V)$, and $\mathbb{R} \oplus$ $\operatorname{coker}(f)$. But there are obvious identifications

$$
\mathbb{R} \oplus \operatorname{coker}(f) \cong f(\mathbb{R}) \oplus \operatorname{coker}(f) \cong \operatorname{coker}(f \mid V) .
$$

\section{POLYNOMIAL FUNCTORS}

We denote by $\mathscr{E}$ the category of continuous functors from $\mathscr{J}$ to unbased spaces. We will switch rather frequently between $\mathscr{E}$ and $\mathscr{E}_{0}$ ("unbased setting" and "based setting"). The unbased setting is considered to be more general, in view of the forgetful functor from $\mathscr{E}$ to $\mathscr{E}_{0}$.

5.1. Definition. An object $E$ in $\mathscr{E}$ is polynomial of degree $\leq n$ if, for every $V$ in $\mathscr{J}_{0}$, the canonical map

$$
\rho: E(V) \longrightarrow \operatorname{holim}_{0 \neq U \subset \mathbb{R}^{n+1}} E(U \oplus V)
$$

is a homotopy equivalence.

Comments. The nonzero linear subspaces $U \subset \mathbb{R}^{n+1}$ form a poset $\mathscr{D}$ where $T \leq U$ means $T \subset U$. (We used the opposite convention in Theorem 4.1.) The rule $U \mapsto E(U \oplus V)$ is then a covariant functor. Again, this has some continuity properties, so we use a topological version of the homotopy limit. According to the standard definition, a point in the homotopy limit would be a rule associating to every functor

$$
G:[n] \longrightarrow \mathscr{D}
$$

a map

$$
f_{G}: \triangle^{n} \longrightarrow E(G(n) \oplus V),
$$

in such a way that $f_{G e}=f_{G} \cdot e_{*}$ for every monotone $e:[m] \rightarrow[n]$. In the present circumstances, we want $f_{G}$ to depend continuously on $G$. To make sense of that, note that if $G$ is close to $H$, say, then we can choose $\omega \in O(n+1)$ close to the identity, such that $\omega G=H$. Then we require that $\omega_{*} f_{G}$ fronı $\Delta^{n}$ to $E(H(n) \oplus V)$ be close to $f_{H}$.

Note the similarity of Definition 5.1 with [Go2, Definition 3.1], and note that the defining property is an extrapolation property. From this point of view it is plausible, but not obvious, that a functor which is polynomial of degree $\leq n$ is also polynomial of degree $\leq n+1$. We shall prove this in Proposition 5.4. 
Recall from section 4 the sphere bundle

$$
S \gamma_{n+1}(V, W) \stackrel{p}{\longrightarrow} \operatorname{mor}(V, W) .
$$

We fix $V$ and vary $W$ to get a natural transformation

$$
S \gamma_{n+1}(V,-) \longrightarrow \operatorname{mor}(V,-) \text {. }
$$

By composition with this one, we then have, for any $E$ in $\mathscr{E}$, a map

$$
p^{*}: \operatorname{nat}(\operatorname{mor}(V,-), E) \longrightarrow \operatorname{nat}\left(S \gamma_{n+1}(V,-), E\right) \text {. }
$$

This map appears in the next proposition; we identify its domain with $E(V)$ by the Yoneda lemma.

5.2. Proposition. A functor $E$ in $\mathscr{E}$ is polynomial of degree $\leq n$ if and only if

$$
p^{*}: E(V) \longrightarrow \operatorname{nat}\left(S \gamma_{n+1}(V,-), E\right)
$$

is a homotopy equivalence for all $V$.

Proof. We begin with a warm-up exercise about mapping spaces and homotopy (co-)limits. Let $X$ be a functor from a small category $\mathscr{J}$ to the category of Kelley spaces (see $[\mathrm{MaL}]$, and let $Y$ be a space. Then, by inspection,

$$
\operatorname{map}(\text { hocolim } X, Y) \cong \operatorname{holim} Y^{X}
$$

where $Y^{X}$ is the functor on $\mathscr{I}^{o p}$ sending $C$ to $\operatorname{map}(X(C), Y)$, the mapping space with the compact-open topology. We will use this remark in a rather generalized situation where $\mathscr{I}$ comes with a topology, and the functor $X$ has continuity properties and is from $\mathscr{F}$ to $\mathscr{E}$. Objects in $\mathscr{E}$ should be regarded as spaces parametrized by $\mathscr{J}$. Homotopy (co-)limits are modified in the usual way to reflect the continuity properties of $X$.

Here we go: $\mathscr{I}=\mathscr{D}$ is the poset of nonzero linear subspaces of $\mathbb{R}^{n+1}$, ordered so that $T \leq U$ means $U \subset T$, and $X$ is given by

$$
X(U)=\operatorname{mor}(U \oplus V,-) \text {. }
$$

Then

$$
\begin{aligned}
\operatorname{nat}\left(S \gamma_{n+1}(V,-), E\right) & \cong \operatorname{nat}(\operatorname{hocolim} X, E) \quad \text { by } 4.2 \\
& \cong \operatorname{holim} \operatorname{nat}(X(U), E) \quad \text { by warm-up and inspection } \\
& \cong \operatorname{holim} E(U \oplus V) \quad \text { by Yoneda. }
\end{aligned}
$$

These are homeomorphisms of spaces "under" $E(V)$, which means: spaces equipped with a map from $E(V)$.

5.3. Proposition. For any $E$ in $\mathscr{E}_{0}$ and any $V$, the sequence

$$
E^{(n+1)}(V) \stackrel{u}{\longrightarrow} E(V) \stackrel{\rho}{\longrightarrow} \operatorname{holim}_{0 \neq U \subset \mathbb{R}^{n+1}} E(U \oplus V)
$$

is a fibration sequence up to homotopy. Hence $E^{(n+1)}$ vanishes if $E$ is polynomial of degree $\leq n$.

Proof. By Proposition 5.2, we may replace $\rho$ by

$$
p^{*}: E(V) \longrightarrow \operatorname{nat}_{0}\left(S \gamma_{n+1}(V,-)_{+}, E\right) \text {. }
$$


We defined $E^{(n+1)}$ by

$$
E^{(n+1)}(V)=\operatorname{nat}_{0}\left(\operatorname{mor}_{n+1}(V,-), E\right) .
$$

Now $\operatorname{mor}_{n+1}(V, W)$ is (simply by definition) the reduced mapping cone of the bundle projection

$$
S \gamma_{n+1}(V, W)_{+} \longrightarrow \operatorname{mor}(V, W)_{+}=\operatorname{mor}_{0}(V, W),
$$

and the claim follows.

5.4. Proposition. If $E$ in $\mathscr{E}$ is polynomial of degree $\leq n-1$, then it is polynomial of degree $\leq n$.

Proof. We will use the following terminology: A morphism

$$
f: C \rightarrow D
$$

in $\mathscr{E}_{0}$ is an E-substitution if the induced map

$$
f^{*}: \operatorname{nat}(D, E) \longrightarrow \operatorname{nat}(C, E)
$$

is a homotopy equivalence. (In general, this is not a very good notion because spaces of natural transformations can be pathological. We only use it in this proof, in very special cases where there is no pathology.)

Fixing $V$ and using Proposition 5.2, we can assume that the bundle projection$$
S \gamma_{n}(\mathbb{R} \oplus V,-) \longrightarrow \operatorname{mor}(\mathbb{R} \oplus V,-)
$$

is an $E$-substitution, and we must show that the inclusion

$$
S \gamma_{n}(V,-) \hookrightarrow S \gamma_{n+1}(V,-)
$$

is an $E$-substitution. We note first that $\gamma_{n+1}(V,-)$ is the Whitney sum of $\gamma_{n}(V,-)$ with $\gamma_{1}(V,-)$. Writing $\otimes$ for fiberwise products (over the Stiefel manifold $\operatorname{mor}(V,-)$ in this case), we can therefore identify $S \gamma_{n+1}(V,-)$ with the homotopy pushout (= double mapping cylinder) of

$$
S \gamma_{n}(V,-) \stackrel{p_{1}}{\longleftarrow} S \gamma_{n}(V,-) \otimes S \gamma_{1}(V,-) \stackrel{p_{2}}{\longrightarrow} S \gamma_{1}(V,-)
$$

where $p_{1}$ and $p_{2}$ are the projections. Now the target of $p_{2}$ can be identified with the Stiefel manifold $\operatorname{mor}(\mathbb{R} \oplus V,-)$, and in fact the map $p_{2}$ itself is just the bundle projection of the sphere bundle made from the vector bundle $\operatorname{res}^{*} \gamma_{n}(V,-)$ in Proposition 4.3. Using Proposition 4.3, we conclude that $S \gamma_{n+1}(V,-)$ is the homotopy pushout of a diagram

$$
S \gamma_{n}(V,-) \longleftarrow S\left(\varepsilon^{n} \oplus \gamma_{n}(\mathbb{R} \oplus V,-)\right) \longrightarrow \operatorname{mor}(\mathbb{R} \oplus V,-) .
$$

We conclude further that $(\$)$ is an $E$-substitution if and only if the bundle projection (formerly $p_{2}$ )

$$
S\left(\varepsilon^{n} \oplus \gamma_{n}(\mathbb{R} \oplus V,-)\right) \longrightarrow \operatorname{mor}(\mathbb{R} \oplus V,-)
$$

is an $E$-substitution. Once more we note that the unit sphere bundle of a Whitney sum of vector bundles is the fiberwise join of the unit sphere bundles of the Whitney summands. Hence the domain in $(\diamond)$ is the homotopy pushout of a diagram

$$
S^{n-1} \times \operatorname{mor}(\mathbb{R} \oplus V,-) \stackrel{q}{\longleftarrow} S^{n-1} \times S \gamma_{n}(\mathbb{R} \oplus V,-) \longrightarrow S \gamma_{n}(\mathbb{R} \oplus V,-) .
$$


The left-hand arrow $q$ is an $E$-substitution by our assumption on (\$) . Consequently the inclusion map

$$
S \gamma_{n}(\mathbb{R} \oplus V,-) \hookrightarrow S\left(\varepsilon^{n} \oplus \gamma_{n}(\mathbb{R} \oplus V,-)\right)
$$

is an $E$-substitution. It follows that $(\diamond)$ is an $E$-substitution if (\$) is an $E$-substitution, which it is by assumption.

5.5. Lemma. Let $g: E \rightarrow F$ be a morphism in $\mathscr{E}_{0}$, assume that $E$ is polynomial of degree $\leq n$, and that $F^{(n+1)}$ vanishes. Then the functor

$$
V \longmapsto \text { hofiber }[E(V) \stackrel{g}{\rightarrow} F(V)]
$$

is also polynomial of degree $\leq n$.

Proof. This is obvious from Propositions 5.2 and 5.3.

5.6. Corollary. If $F$ in $\mathscr{E}_{0}$ is such that $F^{(n+1)}$ vanishes, then the functor

$$
V \longmapsto \Omega(F(V))
$$

is polynomial of degree $\leq n$.

Proof. Apply Lemma 5.5 with $E$ equal to zero.

5.7. Example. Let $\boldsymbol{\theta}$ be a spectrum with an action of $O(n)$, and define $F$ by

$$
F(V):=\Omega^{\infty}\left[\left((n V)^{c} \wedge \boldsymbol{\theta}\right)_{h O(n)}\right]
$$

where the subscript $h O(n)$ indicates a homotopy orbit spectrum (=Borel construction). The group $O(n)$ acts diagonally on the smash product (it acts on $n V$ since $\left.n V=\mathbb{R}^{n} \otimes V\right)$. We shall see that $F$ is polynomial of degree $n$ if $\boldsymbol{\theta}$ is not contractible.

By Corollary 5.6 it suffices to show that $F^{(n+1)}$ vanishes (because $F$ has a delooping which is another functor of the same type). We will do a little more than that. Namely, we will "identify" the sequence of derivatives

$$
F^{(n)} \stackrel{u}{\rightarrow} F^{(n-1)} \stackrel{u}{\rightarrow} F^{(n-2)} \stackrel{u}{\rightarrow} \cdots \quad \ldots \stackrel{u}{\rightarrow} F^{(1)} \stackrel{u}{\rightarrow} F
$$

with another sequence

$$
\begin{gathered}
F[n] \hookrightarrow F[n-1] \hookrightarrow F[n-2] \hookrightarrow \cdots \quad \cdots \hookrightarrow F[1] \hookrightarrow F, \\
F[i](V):=\Omega^{\infty}\left[\left((n V)^{c} \wedge \boldsymbol{\theta}\right)_{h O(n-i)}\right] .
\end{gathered}
$$

Here $O(n-i)$ is the subgroup of $O(n)$ consisting of thoses elements which fix the first $i$ coordinates. Each $F[i]$ is an object in $\mathscr{E}_{i}$. Indeed,

$$
\sigma:(i V)^{c} \wedge F[i](W) \longrightarrow F[i](V \oplus W)
$$

can be defined as an inclusion map,

$$
\begin{aligned}
(i V)^{c} \wedge \Omega^{\infty}\left[\left((n W)^{c} \wedge \boldsymbol{\theta}\right)_{h O(n-i)}\right] & \hookrightarrow \Omega^{\infty}\left[\left((i V)^{c} \wedge(n W)^{c} \wedge \boldsymbol{\theta}\right)_{h O(n-i)}\right] \\
& \hookrightarrow \Omega^{\infty}\left[\left((n(V \oplus W))^{c} \wedge \boldsymbol{\theta}\right)_{h O(n-i)}\right] .
\end{aligned}
$$

In particular, $F[n]$ is a stable object in $\mathscr{E}_{n}$ (see Example 2.3). If it can be "identified" with $F(n)$, then $F(n+1)$ must vanish.

To make the desired "identification" of $F[i]$ with $F^{(i)}$, we will simply establish the following claim. The inclusion map

$$
F[i+1] \hookrightarrow F[i],
$$


which is really a morphism

in $\mathscr{E}_{i}$, has an adjoint

$$
\operatorname{res}_{i}^{i+1} F[i+1] \longrightarrow F[i]
$$

$$
F[i+1] \longrightarrow F[i]^{(1)}
$$

which is an equivalence (see beginning of section 2).

To establish this claim, we use Proposition 2.2. Accordingly, $F[i]^{(1)}(V)$ is the homotopy fiber of

$$
\sigma^{a d}: F[i](V) \longrightarrow \Omega^{i} F[i](\mathbb{R} \oplus V) .
$$

In our case this is homotopy equivalent to $\Omega^{\infty+i+1}$ of the cofiber of the inclusion

$$
\left[(i \mathbb{R} \oplus n V)^{c} \wedge \boldsymbol{\Theta}\right]_{h O(n-i)} \hookrightarrow\left[(n(\mathbb{R} \oplus V))^{c} \wedge \boldsymbol{\Theta}\right]_{h O(n-i)} .
$$

It is also homotopy equivalent to $\Omega^{\infty+1}$ of the cofiber of

$$
\left[(n V)^{c} \wedge \boldsymbol{\Theta}\right]_{h O(n-i)} \hookrightarrow\left[\left(\mathbb{R}^{n-i} \oplus n V\right)^{c} \wedge \boldsymbol{\Theta}\right]_{h O(n-i)} .
$$

which is just $\Omega^{\infty} \Sigma$ of

$$
\left[S_{+}^{n-i-1} \wedge(n V)^{c} \wedge \boldsymbol{\Theta}\right]_{h O(n-i)} .
$$

Now for any spectrum $\Psi$ with an action of $O(k)$, where $k>0$, we have

$$
\left[S_{+}^{k-1} \wedge \Psi\right]_{h O(k)} \simeq \Psi_{h O(k-1)}
$$

using the diagonal action on the wedge. Applying this to ( ), we conclude finally that

$$
F[i]^{(1)}(V) \simeq \Omega^{\infty}\left[\left((n V)^{c} \wedge \boldsymbol{\Theta}\right)_{h O(n-i-1)}\right]=F[i+1](V) .
$$

It is not really difficult to check that this "abstract" homotopy equivalence agrees with the map labelled (

5.7. Example [bis]. The calculation of the derivatives of $F$ also shows what their associated spectra are. Namely,

$$
\begin{aligned}
& \mathbf{\Theta} F^{(i)} \simeq \boldsymbol{\Theta} \quad \text { for } i=n, \\
& \mathbf{\Theta} F^{(i)} \simeq * \quad \text { for } i \neq n, i>0 .
\end{aligned}
$$

The cases $i>n$ and $i=n$ are obvious. For $0<i<n$, observe that

$$
\boldsymbol{\theta} F^{(i)} \simeq \boldsymbol{\theta} F[i] \simeq\left[\boldsymbol{\Theta}\left(\operatorname{res}_{i}^{n} F[n]\right)\right]_{h O(n-i)}
$$

and that $\boldsymbol{\Theta}\left(\operatorname{res}_{i}^{n} D\right)$ is contractible for any $D$ in $\mathscr{E}_{n}$.

5.8. Example. For any $U$ and any $i$ and any $k>0$, the functor $F$ given by

$$
V \longmapsto Q\left(\Sigma^{i} \operatorname{mor}_{k}(U, V)\right)
$$

is polynomial of degree $\leq k+\operatorname{dim}(U)$.

Proof. By Corollary 5.6 again, it suffices to show that $F^{(k+d+1)}$ vanishes where $d=\operatorname{dim}(U)$. We can assume $U=\mathbb{R}^{d}$. Using Lemma 5.5 and Proposition 1.2, we can reduce to the case $d=0$ (by induction, at the price of increasing $k$ ). But $d=0$ means $U=0$, which means

$$
F(V)=Q\left(\Sigma^{i}(k V)^{c}\right) .
$$

This case is covered by Example 5.7. 
Polynomial functors are determined by their behaviour at infinity, i.e., by their behaviour on vector spaces $V$ of very large dimension. To make this precise, let us fix $n \geq 0$ and let

$$
\tau_{n}: \mathscr{E} \longrightarrow \mathscr{E}
$$

be the functor such that

$$
\left(\tau_{n} E\right)(V):=\operatorname{holim}_{0 \neq U \subset \mathbb{R}^{n+1}} E(U \oplus V) .
$$

The canonical map from $E(V)$ to $\left(\tau_{n} E\right)(V)$ is natural in $V$, so it defines a morphism

$$
\rho=\rho_{E}: E \longrightarrow \tau_{n} E .
$$

If $E$ is polynomial of degree $\leq n$, then all morphisms in the diagram

$$
E \stackrel{\rho}{\longrightarrow} \tau_{n} E \stackrel{\rho}{\longrightarrow} \tau_{n} \tau_{n} E \stackrel{\rho}{\longrightarrow} \cdots
$$

are equivalences. But it is obvious that $\left(\tau_{n}\right)^{k} E$ only depends on the behaviour of $E$ on vector spaces $V$ of dimension $\geq k$ (and morphisms between such).

For arbitrary $F$ in $\mathscr{E}_{0}$, the space

$$
F\left(\mathbb{R}^{\infty}\right):=\underset{i}{\operatorname{hocolim}} F\left(\mathbb{R}^{i}\right)
$$

and the spectra $\mathbf{\Theta} F^{(1)}, \boldsymbol{\Theta} F^{(2)}, \boldsymbol{\Theta} F^{(3)}, \ldots$ are determined up to homotopy equivalence by the behaviour of $F$ at infinity. When $F$ is polynomial, we can expect them to have a strong influence on $F(V)$ for all $V$.

5.9. Definition. An object $F$ in $\mathscr{E}$ is connected at infinity if $F\left(\mathbb{R}^{\infty}\right)$ is connected.

Suppose now that $F$ in $\mathscr{E}_{0}$ is polynomial of degree $\leq n$ and connected at infinity. Let $F_{b}$ be the subfunctor of $F$ given by

$$
F_{b}(V)=\text { base point component of } F(V) .
$$

Claim. $F_{b}$ determines $F$, up to equivalence.

Proof. We replace $F$ by the homotopy colimit of

$$
F \stackrel{\rho}{\longrightarrow} \tau_{n} F \stackrel{\rho}{\longrightarrow} \tau_{n} \tau_{n} F \stackrel{\rho}{\longrightarrow} \cdots .
$$

This contains $F$, and the inclusion is an equivalence. On the other hand, it also contains the homotopy colimit of

$$
F_{b} \stackrel{\rho}{\longrightarrow} \tau_{n}\left(F_{b}\right) \stackrel{\rho}{\longrightarrow} \tau_{n} \tau_{n}\left(F_{b}\right) \stackrel{\rho}{\longrightarrow} \cdots .
$$

It remains to show that the inclusion

$$
l: \underset{k}{\operatorname{hocolim}}\left(\tau_{n}\right)^{k} F_{b}(V) \longrightarrow \underset{k}{\operatorname{hocolim}}\left(\tau_{n}\right)^{k} F(V)
$$

is a homotopy equivalence for every $V$. (Equivalently, we must show that all its homotopy fibers are contractible.) Firstly, each homotopy fiber of $l$ is empty or contractible, since

$$
\left(\tau_{n}\right)^{k} F_{b}(V) \hookrightarrow\left(\tau_{n}\right)^{k} F(V)
$$

is the inclusion of a union of connected components, for all $k$. Secondly, we can show that no empty fibers occur: Fix a connected component $C$ in the 
codomain of $l$ (which is homotopy equivalent to $F(V)$ ). Choose a $k \geq 0$ such that the corresponding component $C^{\prime}=C \cap F(V)$ of $F(V)$ maps to the base point component of $F\left(\mathbb{R}^{k} \oplus V\right)$ under the inclusion-induced morphism

$$
F(V) \longrightarrow F\left(\mathbb{R}^{k} \oplus V\right) \text {. }
$$

(This is possible because $F$ is connected at infinity.) Then the image of $C^{\prime}$ in $\left(\tau_{n}\right)^{k} F(V)$ is contained in $\left(\tau_{n}\right)^{k} F_{b}(V)$, so the image of $l$ has nonempty intersection with $C$.

We obtain a very useful corollary.

5.10. Proposition. Let $g: E \rightarrow F$ be a morphism in $\mathscr{E}_{0}$ such that

$$
\text { hofiber }[E(V) \stackrel{g}{\longrightarrow} F(V)]
$$

(the homotopy fiber over the base point) is contractible for all $V$. Assume that $F$ is connected at infinity, and that $E$ and $F$ are polynomial of degree $\leq n$. Then $g$ is an equivalence.

Proof. The assumption on $g$ means that $g_{b}: E_{b} \rightarrow F_{b}$ is an equivalence.

5.11. Lemma. If $E$ in $\mathscr{E}$ is polynomial of degree $\leq m$, then $\tau_{n} E$ is also polynomial of degree $\leq m$, for all $n \geq 0$.

Proof. We have to show that the canonical map

$$
\tau_{n} E(V)=\operatorname{holim}_{0 \neq U \subset \mathbb{R}^{n+1}} E(U \oplus V) \longrightarrow \operatorname{holim}_{0 \neq W \subset \mathbb{R}^{m+1}} \operatorname{holim}_{0 \neq U \subset \mathbb{R}^{n+1}} E(W \oplus U \oplus V)
$$

is a homotopy equivalence, for any $V$. Rewrite the codomain as

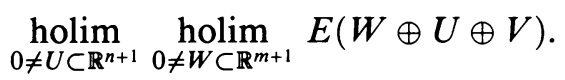

Use the assumption on $E$ and the well-known homotopy invariance of homotopy limits $[\mathrm{BK}]$ to complete the proof.

5.12. Corollary. If $E$ is in $\mathscr{E}_{0}$ is polynomial of degree $\leq m$, then $E^{(m)}$ is stable (as in Example 2.3).

Proof. For $m=0$, this is obvious. When $m>0$, Proposition 5.3 tells us that $E^{(m+1)}$ vanishes. Then the homotopy fiber of

$$
\sigma^{a d}: E^{(m)}(V) \longrightarrow \Omega^{m} E^{(m)}(\mathbb{R} \oplus V)
$$

also vanishes, since it is equal to $E^{(m+1)}(V)$ by Proposition 2.2. We claim now that the functors

$$
V \mapsto E^{(m)}(V), \quad V \mapsto \Omega^{m} E^{(m)}(\mathbb{R} \oplus V)
$$

(as functors on $\mathscr{J}_{0}$ ) are polynomial of degree $\leq m$, and connected at infinity. If so, we can apply Proposition 5.10 to complete the proof. Now the first of these functors is polynomial of degree $\leq m$ by Lemma 5.11 and Lemma 5.5, since $E^{(m)}(V)$ is the homotopy fiber of the canonical map

$$
E(V) \longrightarrow\left(\tau_{m-1} E\right)(V) .
$$

The second of the two functors is then also polynomial of degree $\leq m$ by Corollary 5.6. Both functors are connected at infinity-and even contractible at infinity-because they are restrictions of functors belonging to $\mathscr{E}_{m}$. 
5.13. Corollary. Let $g: E \rightarrow F$ be a morphism in $\mathscr{E}_{0}$ such that the maps

$$
\begin{aligned}
& g_{*}: E\left(\mathbb{R}^{\infty}\right) \longrightarrow F\left(\mathbb{R}^{\infty}\right), \\
& g_{*}: \mathbf{\theta} E^{(i)} \longrightarrow \boldsymbol{\theta} F^{(i)} \quad \text { for } 1 \leq i \leq m
\end{aligned}
$$

are homotopy equivalences. Assume that $E$ and $F$ are polynomial of degree $\leq m$, and that $F$ is connected at infinity. Then $g$ is an equivalence.

Proof. By Proposition 5.10 it is sufficient to show that the functor $D$ given by

$$
D(V):=\text { hofiber }[E(V) \stackrel{g}{\longrightarrow} F(V)]
$$

vanishes. Lemma 5.5 shows that $D$ is polynomial of degree $\leq m$. Let $k \geq 0$ be the least integer such that $D$ is polynomial of degree $\leq k$. Assume first that $k>0$. Then $D^{(k)}$ is stable by Corollary 5.12 . Consequently

$$
D^{(k)}(V) \simeq \Omega^{\infty}\left((k V)^{c} \wedge \boldsymbol{\theta} D^{(k)}\right),
$$

where $\mathbf{\theta} D^{(k)}$ is the homotopy fiber of

$$
g_{*}: \mathbf{\theta} E^{(k)} \longrightarrow \boldsymbol{\theta} F^{(k)} .
$$

But this homotopy fiber is contractible by our assumptions, so $D^{(k)}$ must vanish. Now $D^{(k)}(V)$ is also the homotopy fiber of the canonical map

$$
D(V) \longrightarrow\left(\tau_{k-1} D\right)(V)
$$

in the notation introduced just before Definition 5.9. By Lemma 5.11, the functor $\tau_{k-1} D$ is also polynomial of degree $\leq k$. Consequently, by Proposition 5.10 and Lemma 5.14 just below, the map $(\nabla)$ is a homotopy equivalence for all $V$. This means that $D$ is polynomial of degree $\leq k-1$, in contradiction with the minimality assumption on $k$. We conclude that $D$ must be polynomial of degree 0 , which implies that

$$
D(V) \simeq D\left(\mathbb{R}^{\infty}\right)
$$

for all $V$. But $D\left(\mathbb{R}^{\infty}\right)$ is the homotopy fiber of

$$
g_{*}: E\left(\mathbb{R}^{\infty}\right) \longrightarrow F\left(\mathbb{R}^{\infty}\right),
$$

which is contractible by assumption.

5.14. Lemma. For any $D$ in $\mathscr{E}$ and any $q \geq 0$, the canonical map

$$
D\left(\mathbb{R}^{\infty}\right) \longrightarrow\left(\tau_{q} D\right)\left(\mathbb{R}^{\infty}\right)
$$

is a homotopy equivalence. Hence if $D$ is connected at infinity, then so is $\tau_{q} D$. Proof. The idea is that the codomain of our map is a homotopy colimit of homotopy limits, and we can try to express it as a homotopy limit of homotopy colimits. A more detailed argument follows. Write $U$ for nonzero linear subspaces of $\mathbb{R}^{q+1}$, and $n$ for positive integers, and $\left.\simeq\right\rangle,\langle\simeq$ for homotopy 
equivalences going in the direction indicated. The codomain is

$$
\begin{aligned}
& \text { hocolim holim } D\left(U \oplus \mathbb{R}^{n}\right)\left\langle\simeq \underset{n}{\operatorname{hocolim}} \underset{U}{\operatorname{holim}} \underset{j \leq n}{\operatorname{hocolim}} D\left(U \oplus \mathbb{R}^{j}\right)\right. \\
& \simeq \text { colim } \underset{n}{\operatorname{holim}} \underset{j \leq n}{\operatorname{hocolim}} D\left(U \oplus \mathbb{R}^{j}\right) \\
& \cong \underset{U}{\operatorname{holim}} \underset{j \leq \infty}{\operatorname{hocolim}} D\left(U \oplus \mathbb{R}^{j}\right) \\
& \left\langle\simeq \underset{U}{\operatorname{holim}} \operatorname{hocolim} D\left(\mathbb{R}^{j}\right)\right. \\
& =\underset{U}{\operatorname{holim}} D\left(\mathbb{R}^{\infty}\right) \\
& \left.\simeq \mathbb{R}^{\infty}\right) \text {. }
\end{aligned}
$$

The important thing is the homeomorphism labelled " 1 ", where we use the fact that the nerve of the poset (better: pospace) of all nonzero linear subspaces $U \subset \mathbb{R}^{q+1}$ is compact. For the homotopy equivalence labelled " 2 ", use the contractibility of the nerve (the poset has a maximal element). For the remaining homotopy equivalences, use the homotopy invariance of homotopy limits and homotopy colimits.

We conclude that domain and codomain of the map under investigation are abstractly homotopy equivalent. Inspection reveals that the homotopy equivalence is the map itself.

We will use a half-hearted approach to generalize Corollary 5.13 to the unbased setting. Namely, we will allow base points to vary to some extent. For any $U$ in $\mathscr{J}$, define categories $\mathscr{I}_{m}\langle U\rangle \subset \mathscr{J}_{m}$ as follows. Objects are objects $V$ of $\mathcal{J}$ with a morphism $f$ from $U$ to $V$ in $\mathscr{J}$. A morphism from $(V, f)$ to $(W, g)$ in $\mathscr{J}_{m}\langle U\rangle$ is a morphism $e \in \operatorname{mor}_{m}(V, W)$ making the appropriate triangle commute. To a continuous pointed functor $F$ from $\mathscr{J}_{m}\langle U\rangle$ to pointed spaces, we associate an ordinary spectrum $\boldsymbol{\theta} F$ with $m k$-th term $\Omega^{m U} F\left(\mathbb{R}^{k} \oplus U, f\right)$ where $f$ is the inclusion of the direct summand $U$.

Given $E$ in $\mathscr{E}$, and $U$ in $\mathscr{J}$ and $x \in E(U)$, we construct a functor $E_{x}$ from $\mathscr{J}_{0}\langle U\rangle$ to pointed spaces by $E_{x}(V, f):=E(V)$. We have a base point $f_{*}(x)$ in $E(V)=E_{x}(V, f)$. Hence we can define $E_{x}^{(m)}$, the $m$-th derivative of $E_{x}$, as a functor on $\mathscr{J}_{m}\langle U\rangle$. It is the right Kan extension of $E_{x}$. It gives us an ordinary spectrum $\boldsymbol{\theta} E_{x}^{(m)}$. The good thing about this spectrum is that it varies nicely with $x:$ A morphism $c: U \rightarrow U^{\prime}$ in $\mathscr{J}$ induces

$$
\boldsymbol{\theta} E_{x}^{(m)} \stackrel{\simeq}{\longrightarrow} E_{c x}^{(m)} \quad\left(x \in E(U), c x=c_{*}(x) \in E\left(U^{\prime}\right)\right) .
$$

Hence it is possible to write without too much ambiguity

$$
\boldsymbol{\theta} E_{x}^{(m)} \quad \text { for any } x \in E\left(\mathbb{R}^{\infty}\right) \text {. }
$$

The homotopy type of $\boldsymbol{\theta} E_{x}^{(m)}$ depends only on the connected component of $x$ in $E\left(\mathbb{R}^{\infty}\right)$.

5.15. Theorem. Let $g: E \rightarrow F$ be a morphism in $\mathscr{E}$ such that the maps

$$
\begin{aligned}
& g_{*}: E\left(\mathbb{R}^{\infty}\right) \longrightarrow F\left(\mathbb{R}^{\infty}\right), \\
& g_{*}: \boldsymbol{\Theta} E_{x}^{(i)} \longrightarrow \boldsymbol{\Theta} F_{g x}^{(i)} \quad \text { for } 1 \leq i \leq m, \text { any } x \in E\left(\mathbb{R}^{\infty}\right)
\end{aligned}
$$


are homotopy equivalences. Assume that $E$ and $F$ are polynomial of degree $\leq m$. Then $g$ is an equivalence.

Proof. Both $E$ and $F$ can be written in a unique way as coproducts of functors which are connected at infinity. By Lemma 5.14, each of the summands in these splittings is polynomial of degree $\leq m$. Further, $g$ respects the splitting. Consequently we can assume without loss of generality that $E$ and $F$ are connected at infinity. Our problem is now that $E(0)$ can be empty (otherwise we could just pick a base point there at random, and use Proposition 5.14). However, some $U$ must exist such that $E(U)$ is nonempty. Choose $x \in$ $E(U)$. By our assumptions and by Proposition 5.13, the restriction of $g$ is an equivalence

$$
E_{x} \longrightarrow F_{g x} \text {. }
$$

(Here we apply Corollary 5.13 to polynomial functors on $\mathscr{J}\langle U\rangle$, which is clearly permitted.) This implies at once that

$$
g_{W}: E(W) \longrightarrow F(W)
$$

is a homotopy equivalence for any $W$ such that $\operatorname{dim}(W) \geq \operatorname{dim}(V)$. The principle "polynomial functors are determined by their behaviour at infinity" now shows that $g$ is an equivalence.

5.16. Definition. A morphism $g: E \rightarrow F$ in $\mathscr{E}$ is an approximation of order $m$ if

$$
\begin{aligned}
& g_{*}: E\left(\mathbb{R}^{\infty}\right) \longrightarrow F\left(\mathbb{R}^{\infty}\right), \\
& g_{*}: \boldsymbol{\theta} E_{x}^{(i)} \longrightarrow \boldsymbol{\theta} F_{g x}^{(i)} \quad \text { for } 1 \leq i \leq m, \text { any } x \in E\left(\mathbb{R}^{\infty}\right)
\end{aligned}
$$

are homotopy equivalences.

\section{APPROXIMATION BY POLYNOMIAL FUNCTORS}

Let $\mathscr{A}$ be a full subcategory of another category $\mathscr{B}$. Following [MaL], we say that $\mathscr{A}$ is a reflective subcategory of $\mathscr{B}$ if the inclusion functor $\mathscr{A} \rightarrow \mathscr{B}$ has a left adjoint $T: \mathscr{B} \rightarrow \mathscr{A}$. Put differently: for every object $b$ in $\mathscr{B}$, the comma category $(b \downarrow \mathscr{A})$ has an initial object

$$
\eta_{b}: b \longrightarrow T(b) \text {. }
$$

(The objects of the comma category are morphisms from $b$ to some object in $\mathscr{A}$.) We could say that every object $b$ in $\mathscr{B}$ admits a best approximation (from the right) by an object in $\mathscr{A}$; this is why the notion is interesting here.

6.1. Observation. A full subcategory $\mathscr{A} \subset \mathscr{B}$ is reflective if and only if there exist

(1) a functor $T: \mathscr{B} \rightarrow \mathscr{A}$,

(2) a natural transformation $\eta: 1_{\mathscr{B}} \rightarrow T$ such that

$$
\begin{aligned}
\eta_{a} & : a \rightarrow T(a) \quad(\text { for any } a \text { in } \mathscr{A}) \\
T\left(\eta_{b}\right) & : T(b) \rightarrow T T(b) \quad(\text { for any } b \text { in } \mathscr{B})
\end{aligned}
$$

are isomorphisms. 
Proof. $(\Rightarrow)$ is obvious. For the converse, suppose that $T$ and $\eta$ exist. We claim that $T$ is left adjoint to the inclusion functor. More precisely, every morphism of the form

$$
f: b \longrightarrow a
$$

with $b$ in $\mathscr{B}$ and $a$ in $\mathscr{A}$ has a unique factorization of the form $f=g \eta_{b}$, where $g$ is from $T(b)$ to $a$. Indeed, let

$$
g:=\left(\eta_{a}\right)^{-1} T(f) .
$$

Then $\eta_{a} f=T(f) \eta_{b}=\eta_{a} g \eta_{b}$, so $f=g \eta_{b}$ since $\eta_{a}$ is iso. If $g_{1}$ also satisfies $f=g_{1} \eta_{b}$, then

$$
T(f)=T\left(g_{1}\right) T\left(\eta_{b}\right)=T(g) T\left(\eta_{b}\right),
$$

so that $T\left(g_{1}\right)=T(g)$ since $T\left(\eta_{b}\right)$ is iso. But our assumptions on $\eta$ and $T$ imply that $T$ maps $\operatorname{mor}(T(b), a)$ bijectively to $\operatorname{mor}(T T(b), T(a))$. It follows that $g_{1}=g$.

6.2. Example. Let $\mathscr{B}$ be any category, let

$$
H: \mathscr{B} \longrightarrow \mathscr{B}
$$

be a functor, and let

$$
\rho: 1_{\mathscr{B}} \longrightarrow H
$$

be a natural transformation. Suppose that any functor from the poset of positive integers to $\mathscr{B}$ has a colimit, and that $H$ preserves such colimits. Let $\mathscr{A}$ be the full subcategory of $\mathscr{B}$ whose objects are those $a$ for which

$$
\rho_{a}: a \longrightarrow H(a)
$$

is an isomorphism. Let us try to prove that $\mathscr{A}$ is a reflective subcategory of $\mathscr{B}$. (We will not succeed.)

Attempt. We use Observation 6.1. For any $b$ in $\mathscr{B}$, let $T(b)$ be the colimit of the diagram

$$
b \stackrel{\rho}{\longrightarrow} H(b) \stackrel{H(\rho)}{\longrightarrow} H^{2}(b) \stackrel{H^{2}(\rho)}{\longrightarrow} \cdots
$$

and let $\eta_{b}: b \rightarrow T(b)$ be the obvious morphism. Then $T$ is a functor, and $\eta$ is a natural transformation from $1_{\mathscr{B}}$ to $T$. We must verify that $T$ and $\eta$ satisfy the conditions in Observation 6.1 . The first condition to verify is that $T(b)$ belongs to $\mathscr{A}$ for any $b$. In other words, we must show that

$$
\rho_{T(b)}: T(b) \longrightarrow H T(b)
$$

is iso. Since $H$ preserves certain colimits, and $T(b)$ is such a colimit, we find that $H T(b)$ is (isomorphic to) the colimit of

$$
H(b) \stackrel{H(\rho)}{\longrightarrow} H^{2}(b) \stackrel{H^{2}(\rho)}{\longrightarrow} H^{3}(\rho) \stackrel{H^{3}(\rho)}{\longrightarrow} \cdots
$$

which is "abstractly" isomorphic to $T(b)$. The more obvious isomorphism is from $H T(b)$ to $T(b)$, and happens to be inverse to $\rho_{T(b)}$. This shows that $T(b)$ belongs to $\mathscr{A}$. Further, it is obvious that $\eta_{a}$ is iso for any $a$ in $\mathscr{A}$. Unfortunately, it seems impossible to prove without additional hypotheses that $T\left(\eta_{b}\right)$ is iso for any $b$ in $\mathscr{B}$. We can only hope that it will work out magically in special cases of special interest. 
Let us apply these observations to the following situation: $\mathscr{B}=\mathscr{E}$, the category of continuous functors from $\mathscr{J}$ to unbased spaces, and $\mathscr{A}$ is the full subcategory made up of all objects which are polynomial of degree $\leq n$ for some fixed $n$. In this case it would be unreasonable to expect that $\mathscr{A}$ is a reflective subcategory in the strict sense. However, we have the alternative definition of "reflective subcategory" given by Observation 6.1. We relax it just a little.

6.3. Theorem. For any $n \geq 0$, there exist a functor

$$
T_{n}: \mathscr{E} \longrightarrow \mathscr{E}
$$

taking equivalences to equivalences, and a natural transformation

$$
\eta_{n}: 1 \longrightarrow T_{n}
$$

with the following properties:

(1) $T_{n}(E)$ is polynomial of degree $\leq n$, for all $E$ in $\mathscr{E}$.

(2) if $E$ is already polynomial of degree $\leq n$, then

$$
\eta_{n}: E \longrightarrow T_{n} E
$$

is an equivalence.

(3) For every $E$ in $\mathscr{E}$, the map

$$
T_{n}\left(\eta_{n}\right): T_{n} E \longrightarrow T_{n} T_{n} E
$$

is an equivalence.

Proof. We proceed as in Example 6.2, letting $H=\tau_{n}$ (defined just after Example 5.8) and taking for $\rho$ the canonical transformation. Thus $T_{n} E$ is, by definition, the homotopy colimit of

$$
E \stackrel{\rho}{\longrightarrow} \tau_{n} E \stackrel{\tau_{n}(\rho)}{\longrightarrow}\left(\tau_{n}\right)^{2} E \stackrel{\left(\tau_{n}\right)^{2}(\rho)}{\longrightarrow} \cdots,
$$

and $\eta_{n}: E \rightarrow T_{n} E$ is the evident inclusion. As in Example 6.2, we find that

$$
\rho: T_{n} E \longrightarrow \tau_{n} T_{n} E
$$

is an equivalence for arbitrary $E$, which means that $T_{n} E$ is polynomial of degree $\leq n$. If $E$ is already polynomial of degree $\leq n$, then clearly

$$
\eta_{n}: E \longrightarrow T_{n} E
$$

is an equivalence. It remains to establish property (3). This will (eventually) come out of Theorem 5.15.

Step 1: We note that

$$
\rho: E \longrightarrow \tau_{n} E
$$

is an approximation of order $n$ (Definition 5.16.).

Proof. Lemma 5.14 shows that

$$
\rho_{*}: E\left(\mathbb{R}^{\infty}\right) \longrightarrow\left(\tau_{n} E\right)\left(\mathbb{R}^{\infty}\right)
$$

is a homotopy equivalence. Further, if we pick $j \geq 0$ and $x \in E\left(\mathbb{R}^{j}\right)$, then $\left(\tau_{n}\right)\left(E_{x}\right)$ is the same as $\left(\tau_{n} E\right)_{\rho x}$, and the homotopy fiber (functor) $D_{x}$ of

$$
\rho: E_{x} \longrightarrow\left(\tau_{n} E\right)_{\rho x}
$$


is therefore $E_{x}^{(n+1)}$ by Propositions 5.3 and 5.2. Therefore $D_{x}$ extends from $\mathscr{E}_{0}\left\langle\mathbb{R}^{j}\right\rangle$ to $\mathscr{E}_{n+1}\left\langle\mathbb{R}^{j}\right\rangle$, therefore $\boldsymbol{\Theta} D_{x}^{(i)}$ is contractible for $1 \leq i \leq n$, and therefore

$$
\rho_{*}: \boldsymbol{\theta} E_{x}^{(i)} \longrightarrow \boldsymbol{\Theta} E_{\rho x}^{(i)}
$$

is a homotopy equivalence for $1 \leq i \leq n$. This proves that $\left({ }^{*}\right)$ is an approximation of order $n$.

Step 2: We note that all arrows in the diagram

$$
E \stackrel{\rho}{\longrightarrow} \tau_{n} E \stackrel{\tau_{n}(\rho)}{\longrightarrow}\left(\tau_{n}\right)^{2} E \stackrel{\left(\tau_{n}\right)^{2}(\rho)}{\longrightarrow} \ldots
$$

are approximations of order $n$. We can prove this by induction, using the commutative diagrams

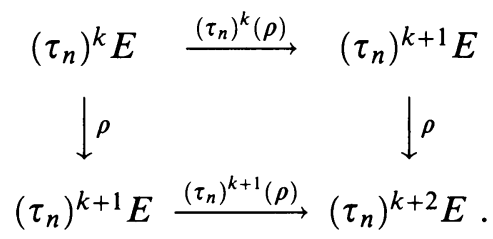

We have just shown that the vertical arrows in the diagram are approximations of order $n$, and if the same is true for the upper horizontal arrow, then it must be true for the lower horizontal arrow as well.

Step 3: We conclude from the last step that

$$
\eta_{n}: E \longrightarrow T_{n} E
$$

is an approximation of order $n$, for any $E$. This shows that three of the four arrows in the commutative diagram

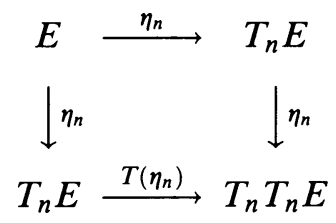

are approximations of order $n$. Therefore

$$
T\left(\eta_{n}\right): T_{n} E \longrightarrow T_{n} T_{n} E
$$

is also an approximation of order $n$. Now Theorem 5.15 implies that $T\left(\eta_{n}\right)$ is an equivalence.

6.3. Theorem [bis]. For any $n \geq 0$ and any $E$ in $\mathscr{E}$, the morphism $\eta_{n}$ from $E$ to $T_{n} E$ is an approximation of order $n$.

6.4. Example. Let $\boldsymbol{\Theta}$ be a spectrum with an action of $O(n)$, where $n>0$, and let $E, F$ in $\mathscr{E}$ be given by

$$
\begin{aligned}
& E(V):=\left[\Omega^{\infty}\left((n V)^{c} \wedge \boldsymbol{\theta}\right)\right]_{h O(n)}, \\
& F(V):=\Omega^{\infty}\left[\left((n V)^{c} \wedge \boldsymbol{\theta}\right)_{h O(n)}\right] .
\end{aligned}
$$

In the definition of $E$, the subscript $h O(n)$ denotes a homotopy orbit space in the pointed category, i.e., the quotient of the homotopy orbit space in the 
unpointed category by $B O(n)$. We know $F$ from Example 5.7, and $E$ is a subfunctor of $F$. Let

$$
l: E \hookrightarrow F
$$

be the inclusion. We shall verify that $T_{n}(l): T_{n} E \longrightarrow T_{n} F$ is an equivalence. This is in effect a calculation of $T_{n} E$, since $\eta_{n}: F \longrightarrow T_{n} F$ is also an equivalence by Example 5.7 and Theorem 6.3, item (2) .

The argument which follows is due to the referee, and it is much simpler than my own. We shall prove more, namely, $T_{2 n-1}(l): T_{2 n-1} E \longrightarrow T_{2 n-1} F$ is an equivalence.

Step 1. By Theorem 5.15 and Theorem 6.3 [bis], it is enough to show that $l: E \rightarrow F$ is an approximation of order $2 n-1$.

Step 2. If the Lie group $G$ acts on a spectrum $\mathbf{X}$ and $\mathbf{X}$ is $k$-connected, $k>0$, then the canonical map from $\left(\Omega^{\infty} \mathbf{X}\right)_{h G}$ to $\Omega^{\infty}\left(\mathbf{X}_{h G}\right)$ is $2 k+1$-connected. Indeed, in the special case where $\mathbf{X}$ is the suspension spectrum of a $k$-connected based $G$-space $Y$, the map in question is the second arrow in

$$
Y_{h G} \hookrightarrow(Q Y)_{h G} \hookrightarrow Q\left(Y_{h G}\right),
$$

and it is $(2 k+1)$-connected because both the first arrow and the composition are. In general $\mathbf{X}$ admits a $(2 k+2)$-connected map $\Sigma^{\infty} Y \rightarrow \mathbf{X}$ from such a suspension spectrum (let $Y=\Omega^{\infty} \mathbf{X}$ ) and this can be used to reduce to the special case.

Step 3. Assume that $\boldsymbol{\theta}$ is $k$-connected for some $k \in \mathbb{Z}$. (Briefly: $\boldsymbol{\theta}$ is bounded below.) Then $l: E(V) \rightarrow F(V)$ is $[2 n \cdot \operatorname{dim}(V)-c]$-connected, where $c$ is a constant depending on $\boldsymbol{\theta}$ but not on $V$. This follows from Step 2. Looking at the homotopy fiber of $l: E(V) \rightarrow F(V)$, as a functor in the variable $V$, we deduce immediately that $l: E \rightarrow F$ is an approximation of order $2 n-1$. (Use the elementary definition of derivatives at infinity from the introduction.)

Step 4. The case of general $\boldsymbol{\theta}$ follows from Step 3 by writing $\boldsymbol{\theta}$ as a homotopy colimit of a sequence of spectra which are bounded below.

\section{HOMOGENEOUS FUNCTORS}

7.1. Definition. An object $F$ in $\mathscr{E}$ is homogeneous of degree $n$ if it is polynomial of degree $\leq n$ and if $T_{n-1} E(V)$ is contractible for all $V$.

We want to "classify" such objects. This is much easier in the setting "with base points", so we start by classifying homogeneous objects $F$ in $\mathscr{E}_{0}$. In fact, we start by classifying their $n$-th derivatives $F^{(n)}$. Corollary 5.12 tells us that $F^{(n)}$ is stable, and Proposition 3.1 tells us that $F^{(n)}$ is symmetric (Definition 3.3). Thus our first task is to classify stable symmetric objects in $\mathscr{E}_{n}$, for any $n \geq 0$.

The case $n=0$ is contained in Example 2.3. We can reformulate the result as follows. Let $\alpha_{0}$ be the rule taking an object $F$, homogeneous of degree 0 , to the pointed space $F\left(\mathbb{R}^{\infty}\right)$. Let $\beta_{0}$ be the rule taking a pointed space $Y$ to the constant functor $V \mapsto Y$, which is homogeneous of degree 0 . There is a natural homotopy equivalence

$$
Y \longrightarrow \alpha_{0} \beta_{0}(Y)
$$


of pointed spaces, and there are equivalences

$$
F \longrightarrow \hat{F} \longleftarrow \beta_{0} \alpha_{0}(F),
$$

where $\hat{F}$ is the functor

$$
V \longmapsto \underset{k}{\operatorname{hocolim}} F\left(\mathbb{R}^{k} \oplus V\right) .
$$

Moreover, $\alpha_{0}$ takes equivalences to homotopy equivalences, and $\beta_{0}$ takes homotopy equivalences to equivalences.

Now take $n>0$, and let $\alpha_{n}$ be the rule taking a symmetric stable object $E$ in $\mathscr{E}_{n}$ to the spectrum with $k$-th term

$$
\Omega^{\infty}\left(S^{k} \wedge \boldsymbol{\theta} E\right) .
$$

This comes with an action of $O(n)$, as described in Example 3.3. Let $\beta_{n}$ be the rule taking a spectrum $\boldsymbol{\Theta}$ with an action of $O(n)$ to the functor

$$
V \longmapsto \Omega^{\infty}\left((n V)^{c} \wedge \boldsymbol{\theta}\right)
$$

We regard this functor as a stable object in $\mathscr{E}_{n}$, as explained in Definition 2.3. We make it into a symmetric object using the diagonal action of $O(n)$ on $(n V)^{c} \wedge \boldsymbol{\Theta}$, for each $V$.

Now $\alpha_{n} \beta_{n}(\boldsymbol{\Theta})$ is the spectrum with $k$-th term

$$
\Omega^{\infty}\left(S^{k} \wedge \boldsymbol{\Theta}^{\sharp}\right),
$$

in the notation of Example 3.3. This contains $\boldsymbol{\Theta}$ since

$$
\boldsymbol{\Theta}_{k} \subset \boldsymbol{\Omega}^{k}\left(S^{k} \wedge \boldsymbol{\Theta}_{k}\right) \subset \Omega^{\infty}\left(S^{k} \wedge \boldsymbol{\Theta}\right)
$$

and $\boldsymbol{\Theta} \subset \boldsymbol{\Theta}^{\sharp}$. The inclusion

$$
\boldsymbol{\Theta} \hookrightarrow \alpha_{n} \beta_{n}(\boldsymbol{\Theta})
$$

is a homotopy equivalence, and is compatible with the $O(n)$-actions.

In the other direction, we observe that $\beta_{n} \alpha_{n}(E)$ is (equivalent to) the functor

$$
V \longmapsto \underset{k, j \rightarrow \infty}{\operatorname{hocolim}} \Omega^{k}\left[(n V)^{c} \wedge \Omega^{n j}\left(S^{k} \wedge E\left(\mathbb{R}^{j}\right)\right)\right] .
$$

(on $\mathscr{J}_{n}$ ). We use the maps

$$
\sigma:(n V)^{c} \wedge E\left(\mathbb{R}^{j}\right) \longrightarrow E\left(V \oplus \mathbb{R}^{j}\right)
$$

to map this to another functor $\hat{E}$ on $\mathscr{J}_{n}$, where

$$
\hat{E}(V):=\underset{k, j \rightarrow \infty}{\operatorname{hocolim}} \Omega^{k+n j}\left(S^{k} \wedge E\left(V \oplus \mathbb{R}^{j}\right)\right) .
$$

This new functor $\hat{E}$ contains $E$ (take $k, j=0$ ). Hence we have equivalences

$$
E \longrightarrow \hat{E} \longleftarrow \beta_{n} \alpha_{n}(E)
$$

of stable symmetric objects in $\mathscr{E}_{n}$.

All this shows that, for $n>0$, classifying stable symmetric objects in $\mathscr{E}_{n}$ up to equivalence amounts to classifying spectra with an action of $O(n)$ up to a suitable notion of equivalence. To summarize the result in a proposition, we introduce some more notation. Let $\mathscr{X}_{n}$ be the category whose objects are the stable symmetric objects in $\mathscr{E}_{n}$, and whose morphisms are the natural transformations $E \rightarrow F$ (respecting symmetry) between such objects. Let $\mathscr{Y}_{n}$ 
be the category whose objects are spectra with an action of $O(n)$, and whose morphisms are maps $\boldsymbol{\theta} \rightarrow \Psi$ compatible with the actions of $O(n)$. The constructions $\alpha_{n}, \beta_{n}$ are simply functors,

$$
\alpha_{n}: \mathscr{X}_{n} \rightarrow \mathscr{Y}_{n}, \quad \beta_{n}: \mathscr{Y}_{n} \rightarrow \mathscr{X}_{n} .
$$

7.2. Proposition. For $E$ in $\mathscr{X}_{n}$ there exists a chain of natural equivalences (in $\mathscr{X}_{n}$ ) relating $E$ to $\beta_{n} \alpha_{n}(E)$. For $\boldsymbol{\theta}$ in $\mathscr{Y}_{n}$, there exists a natural homotopy equivalence (in $\mathscr{Y}_{n}$ ) from $\boldsymbol{\theta}$ to $\alpha_{n} \beta_{n}(\mathbf{\theta})$.

7.3. Theorem. Let $F$ in $\mathscr{E}_{0}$ be homogeneous of degree $n$, where $n>0$. Then $F$ is equivalent to the functor

$$
V \longmapsto \Omega^{\infty}\left[\left((n V)^{c} \wedge \Psi\right)_{h O(n)}\right]
$$

(cf. Example 5.7), where $\boldsymbol{\Psi}=\boldsymbol{\theta}^{\sharp} F^{(n)} \simeq \boldsymbol{\theta} F^{(n)}$, with the $O(n)$-action specified in section 3. Conversely, any functor of the form $(\downarrow)$ is homogeneous of degree $n$.

Proof. Let $E$ in $\mathscr{E}_{0}$ be the functor given by

$$
E(V)=\left(F^{(n)}(V)\right)_{h O(n)}
$$

(use the action of Proposition 3.1, and remember the meaning of the subscript $h O(n)$, as in Example 6.4). This contains $F^{(n)}$, or rather $\operatorname{res}_{0}^{n} F^{(n)}$, as a subfunctor. What we know about $F^{(n)}$ (from Proposition 7.2) implies that

(1) $T_{n} E$ is equivalent to the functor

$$
V \longmapsto \Omega^{\infty}\left[\left((n V)^{c} \wedge \Psi\right)_{h O(n)}\right]
$$

(by Example 6.4), and this is homogeneous of degree $n$ (by Example 5.7);

(2) the adjoint of the composition

$$
\operatorname{res}_{0}^{n}\left(F^{(n)}\right) \hookrightarrow E \stackrel{\eta_{n}}{\longrightarrow} T_{n} E
$$

is an equivalence

$$
F^{(n)} \longrightarrow\left(T_{n} E\right)^{(n)}
$$

(again by Example 5.7).

Recall now (Proposition 3.1) that

$$
u: F^{(n)}(V) \longrightarrow F(V)
$$

is an $O(n)$-map for the trivial action of $O(n)$ on $F(V)$, for all $V$. This implies that

has a factorization as

$$
u: \operatorname{res}_{0}^{n} F^{(n)} \longrightarrow F
$$

$$
\operatorname{res}_{0}^{n} F^{(n)} \hookrightarrow E \stackrel{\widehat{u}}{\longrightarrow} F
$$

We use $\widehat{u}$ to make a commutative diagram

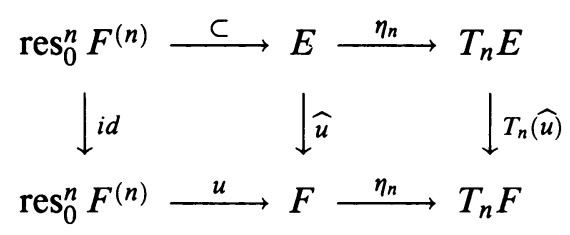


and we proceed to prove that $T_{n}(\widehat{u})$ is an equivalence. (This will complete the proof because $\eta_{n}$ from $F$ to $T_{n} F$ is also an equivalence.) The adjoint of the composite map in the lower row of the diagram is an equivalence

$$
F^{(n)} \longrightarrow\left(T_{n} F\right)^{(n)}
$$

(because the adjoint of $u$ is an equivalence, and $\eta_{n}$ in the lower row is an equivalence). We have already verified that the adjoint of the composite map in the upper row is also an equivalence. Commutativity of the diagram now shows that $T_{n}(\widehat{u})$ induces an equivalence of $n$-th derivatives. But then $T_{n}(\widehat{u})$ must be an equivalence, by Corollary 5.13 .

It is not difficult to formulate and prove a more precise version of theorem 7.3, along the lines of Proposition 7.2. There is a functor

$$
F \mapsto \alpha_{n}\left(F^{(n)}\right),
$$

taking homogeneous functors of degree $n$ to spectra with an action of $O(n)$. There is another functor

$$
\boldsymbol{\theta} \longmapsto\left[V \mapsto \Omega^{\infty}\left[\left((n V)^{c} \wedge \boldsymbol{\theta}\right)_{h O(n)}\right]\right]
$$

taking spectra with an action of $O(n)$ to homogeneous functors of degree $n$. These two functors are inverses of one another up to chains of natural equivalences.

7.4. Lemma. Suppose that $F$ in $\mathscr{E}_{0}$ is homogeneous of degree $n$, where $n>0$. Then the adjoint of

is an equivalence

$$
\eta_{n}: \Sigma F \longrightarrow T_{n}(\Sigma F)
$$

$$
F \longrightarrow \Omega T_{n}(\Sigma F) \text {. }
$$

Explanation and comment. The functor $\Sigma F$ takes $V$ in $\mathscr{J}_{0}$ to the reduced suspension of $F(V)$. The proof of Lemma 7.4 will be very easy once we have a good category of fractions $\mathscr{E}_{0} / \sim$ (made from $\mathscr{E}_{0}$ by inverting all equivalences). To explain what this looks like, I shall borrow a definition and a result from the appendix. An object $D$ in $\mathscr{E}_{0}$ is cofibrant if, for any diagram

$$
D \stackrel{c}{\longrightarrow} F \stackrel{e}{\longleftarrow} \hat{F}
$$

in $\mathscr{E}_{0}$ where $e$ is an equivalence, there exist a morphism

$$
\hat{c}: D \longrightarrow \hat{F}
$$

and a natural homotopy $h$ from $e \cdot \hat{c}$ to $c$. There is a functorial construction giving, for each $E$ in $\mathscr{E}_{0}$, a cofibrant object $E^{\diamond}$ and an equivalence $\varepsilon$ from $E^{\diamond}$ to $E$. Consequently the category of fractions $\mathscr{E}_{0} / \sim$ can be described as follows: Objects are the objects of $\mathscr{E}_{0}$, and a morphism from $E$ to $F$ in $\mathscr{E}_{0} / \sim$ is a homotopy class of morphisms from $E^{\diamond}$ to $F^{\diamond}$ in $\mathscr{E}_{0}$. A morphism $g$ from $E$ to $F$ in $\mathscr{E}_{0}$ determines a morphism from $E$ to $F$ in $\mathscr{E}_{0} / \sim$, namely, the homotopy class of $g^{\diamond}$. If $g$ is an equivalence, then the class of $g^{\diamond}$ is an isomorphism in $\mathscr{E}_{0} / \sim$. We write $[E, F]$ for the set of morphisms from $E$ to $F$ in $\mathscr{E}_{0} / \sim$.

Proof of Lemma 7.4. As an endofunctor of $\mathscr{E}_{0} / \sim$, the functor $T_{n}$ is left adjoint to the inclusion of the full subcategory consisting of all functors which are 
polynomial of degree $\leq n$. Therefore, if $E$ is polynomial of degree $\leq n$, we have

$$
\left[T_{n}(\Sigma F), E\right] \cong[\Sigma F, E] \cong[F, \Omega E] .
$$

If $E$ is polynomial of degree $\leq n-1$, then so is $\Omega E$, and therefore

$$
[F, \Omega E] \cong\left[T_{n-1} F, \Omega E\right]=\{*\} .
$$

It follows that $\left[T_{n}(\Sigma F), E\right]$ is zero if $E$ is polynomial of degree $\leq n-1$. Therefore $T_{n}(\Sigma F)$ must be homogeneous of degree $n$. We see that

$$
F \mapsto T_{n}(\Sigma F)
$$

is an endofunctor of the full subcategory $\mathscr{H}_{n}$ of $\mathscr{E}_{0} / \sim$ consisting of the objects which are homogeneous of degree $n$. It is left adjoint to the endofunctor of $\mathscr{H}_{n}$ given by

$$
F \mapsto \Omega F .
$$

But our classification result for homogeneous functors (Theorem 7.3 and sequel) implies that the second endofunctor is an equivalence of the category $\mathscr{H}_{n}$ with itself. Hence the unit of the adjunction [MaL] must be an isomorphism

$$
F \longrightarrow \Omega T_{n}(\Sigma F) \text {. }
$$

Last not least, we come to the classification of homogeneous objects in $\mathscr{E}$.

Notation. For a space $X$, denote by $\Sigma \natural X$ the unreduced suspension of $X$. For a space $Y$ with two distinguished points (called north pole and south pole), let $\Omega_{\natural} Y$ be the space of paths in $Y$ from north pole to south pole.

7.5. Lemma. Suppose that $F$ in $\mathscr{E}$ is homogeneous of degree $n$, where $n>0$. Then the adjoint of

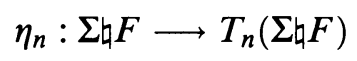

is an equivalence

$$
F \longrightarrow \Omega \natural T_{n}(\Sigma \natural F) .
$$

Proof. As in the proof of 5.15, our problem is that $F(0)$ can be empty. However, for some $U$ in $\mathscr{J}$ the space $F(U)$ will be nonempty: pick a base point $x$ in $F(U)$ at random. Lemma 7.4, applied to the homogeneous functor

$$
(V, f) \mapsto F(V)
$$

from $\mathscr{L}\langle V\rangle$ to pointed spaces, shows that

$$
F(V) \longrightarrow \Omega \natural T_{n}(\Sigma \natural F(V))
$$

is a homotopy equivalence for all $V$ such that $\operatorname{dim}(V) \geq \operatorname{dim} U$. This means that the hypotheses in Theorem 5.15 are satisfied. Now apply the theorem to complete the proof.

Note that $E:=T_{n}(\Sigma \natural F)$ in Lemma 7.5 is an object in $\mathscr{E}_{0}$ (and by the lemma, it is homogeneous of degree $n$ ). Actually, lifting $E$ from $\mathscr{E}$ to $\mathscr{E}_{0}$ amounts to choosing a base point in $E(0)$; here we seem to have two canonical choices, the image of north pole and south pole under

$$
\eta_{n}: \Sigma \natural F(0) \longrightarrow T_{n}(\Sigma \natural F)(0) .
$$


We declare the north pole to be the base point. Now Lemma 7.4 tells us that the functor $F$ is equivalent to the homotopy pullback of

$$
* \hookrightarrow E \stackrel{y}{\longleftarrow} *
$$

where $*$ is the terminal object in $\mathscr{E}$ and $\hookrightarrow$ picks the base point, whereas $y$ picks the south pole. Conversely, given $D$ in $\mathscr{E}_{0}$, homogeneous of degree $n$, and a point $z \in D(0)$, the homotopy pullback of

$$
* \hookrightarrow D \stackrel{z}{\longleftarrow} *
$$

is a homogeneous object of degree $n$ in $\mathscr{E}$. The verification is easy. Combining this with Theorem 7.3, we have a classification of homogeneous objects in $\mathscr{E}$ up to equivalence.

7.6. Example. For any $n>0$ and any $k \geq 0$, there exists an $F$ in $\mathscr{E}$, homogeneous of degree $n$, such that $F\left(\mathbb{R}^{k}\right)$ is empty (hence $F(V)=\varnothing$ whenever $\operatorname{dim}(V) \leq k)$. To construct such an $F$, we start with the projection map

$$
p: \operatorname{mor}\left(\mathbb{R}^{n}, \mathbb{R}^{n+i}\right) \longrightarrow G_{n, i}
$$

(large $i$ ) from the Stiefel manifold of orthonormal $n$-frames in $\mathbb{R}^{n+i}$ to the Grassmann manifold of $n$-planes through the origin in $\mathbb{R}^{n+i}$. Let $\nu$ be the normal bundle of $G_{n, i}$ (in some $\mathbb{R}^{N}$ ) and let $p^{*} \nu$ be its pullback to the Stiefel manifold. Then the Thom spectrum $\mathbf{t}\left(p^{*} \nu\right)$ of $p^{*} \nu$ comes with an evident action of $O(n)$, free in the based sense and with orbit spectrum equal to $\mathbf{t}(\nu)$. Let $\boldsymbol{\theta}=\Omega^{n i} \mathbf{t}\left(p^{*} \nu\right)$. Then

$$
\boldsymbol{\theta}_{h O(n)} \simeq \Omega^{n i} \mathbf{t}(\nu)
$$

which has $\left(G_{n, i}\right)_{+}$as Spanier-Whitehead dual. Let $y$ in $\Omega^{\infty}\left(\boldsymbol{\Theta}_{h O(n)}\right)$ be SWdual to the unit map from $\left(G_{n, i}\right)_{+}$to $\mathbf{S}^{0}$. Then $y$ belongs to $E(0)$, where $E$ is the functor

$$
V \mapsto \Omega^{\infty}\left[\left((n V)^{c} \wedge \boldsymbol{\theta}\right)_{h O(n)}\right]
$$

Let $F$ be the homotopy pullback of

$$
* \hookrightarrow E \stackrel{y}{\longleftarrow} *
$$

(where the left-hand arrow is the standard inclusion). We shall see that the image of $y$ in $E\left(\mathbb{R}^{k}\right)$ is not in the connected component of the base point, so that $F\left(\mathbb{R}^{k}\right)$ must be empty. Since

$$
E\left(\mathbb{R}^{k}\right)=\Omega^{\infty}\left[\left(\left(n \mathbb{R}^{k}\right)^{c} \wedge \boldsymbol{\theta}\right)_{h O(n)}\right]
$$

we can try to calculate the class of $y$ in the spectrum homology $H_{0}(-; \mathbb{Z} / 2)$ of

$$
\left(\left(n \mathbb{R}^{k}\right)^{c} \wedge \boldsymbol{\theta}\right)_{h O(n)}
$$

Under Thom isomorphism and Poincaré duality, this homology group becomes

$$
H^{n k}\left(G_{n, i} ; \mathbb{Z} / 2\right)
$$


and $y$ represents the $k$-fold power of the Euler class of the tautological $n-$ plane bundle on $G_{n, i}$. This will be nonzero if $i$ is sufficiently large.

\section{HOMOGENEOUS MORPHISMS}

8.1. Definition. A morphism $g: E \rightarrow F$ in $\mathscr{E}$ is polynomial of degree $\leq n$ if

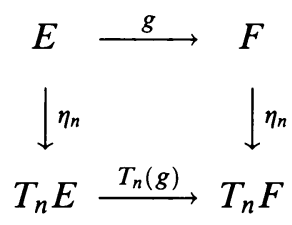

is a homotopy pullback square. If in addition

$$
T_{n-1} g: T_{n-1} E \rightarrow T_{n-1} F
$$

is an equivalence, then $g$ is homogeneous of degree $n$.

Intuitively, a morphism in $\mathscr{E}$ is polynomial of degree $\leq n$, or homogeneous of degree $n$, if its fibers are. In particular, the morphism from $E$ to the zero object is polynomial/homogeneous if and only if $E$ is polynomial/homogeneous. We need Definition 8.1 in order to express a rigidity property of homogeneous objects.

8.2. Theorem. Let $g: E \rightarrow F$ be homogeneous of degree $n$, where $n>0$. Suppose that $F\left(\mathbb{R}^{\infty}\right)$ is contractible, and that there exist $D$ and

$$
s: D \longrightarrow E
$$

in $\mathscr{E}$ such that $g \cdot s$ is an equivalence. Then there exist $G$ and

$$
w: E \longrightarrow G
$$

in $\mathscr{E}$ such that $(g, w)$ from $E$ to $F \times G$ is an equivalence.

Intuitively: if a homogeneous morphism $g$ has a section, and if its codomain is contractible at infinity, then its domain is a product and $g$ is projection to a factor.

Preliminaries. We can make the following additional assumptions:

(1) $D=F$ and $g s$ is the identity;

(2) $g: E(V) \longrightarrow F(V)$ is a fibration for all $V$.

To arrange this, replace $E$ by the homotopy pullback $\hat{E}$ of

$$
D \stackrel{g s}{\longrightarrow} F \stackrel{g}{\longleftarrow} E
$$

and replace $g$ by the projection $\hat{g}$ from $\hat{E}$ to $D$. Then $\hat{g}$ has an evident section $\hat{s}$. If $\hat{w}$ from $\hat{E}$ to $\hat{G}$ can be constructed such that $(\hat{g}, \hat{w})$ is an equivalence from $\hat{E}$ to $D \times \hat{G}$, then let $G$ be the homotopy pushout of

$$
E \stackrel{\simeq}{\simeq} \stackrel{\hat{w}}{\longrightarrow} \hat{G}
$$

and let $w$ be the inclusion of $E$ in $G$.

Proof of Theorem 8.2. For each $V$ and $x \in F(V)$, define a functor $\phi x$ from $\mathscr{L}_{0}\langle V\rangle$ to pointed spaces by

$$
\phi x(W, f):=g^{-1}\left(f_{*}(x)\right) \subset E(W)
$$


where $f_{*}(x) \in F(W)$, and $s f_{*}(x)$ serves as the base point of $\phi x(W, f)$. Define $A$ in $\mathscr{E}$ by

$$
A(V):=\left\{(x, z) \mid x \in F(V), \quad z \in \phi x^{(n)}(V, i d)\right\} .
$$

In words: $A(V)$ is the total space of a fibration over $F(V)$ whose fiber over $x \in F(V)$ is $\phi x^{(n)}(V, i d)$. Here $\phi x^{(n)}$ is the $n$-th derivative of $\phi x$, a functor from $\mathscr{J}_{m}\langle V\rangle$ to pointed spaces. Note that $O(n)$ acts on $A$, by

$$
g \cdot(x, z):=(x, g z) \quad \text { for } g \in O(n) \text { and }(x, z) \in A(V),
$$

and the morphism $\bar{u}: A \rightarrow E$ given by

$$
(x, z) \mapsto u(z) \in \phi x(V, i d) \subset E(V) \quad \text { for }(x, z) \in A(V)
$$

is invariant under this action. (Here $u$ is the universal map from $\phi x^{(n)}$ to $\phi x$.) Hence we also have

$$
\bar{u}_{\text {orb }}: A_{h O(n)} \longrightarrow E
$$

where $A_{h O(n)}(V)$ means $A(V)_{h O(n)}$, and $A(V)_{h O(n)}$ is very sloppy notation for a homotopy orbit construction in the category of retractive spaces over $E(V)$. We shall now verify that $A$ splits up to equivalence as a product of $F$ with something else, and that $A_{h O(n)}$ and $T_{n}\left(A_{h O(n)}\right)$ split similarly. We will then complete the proof by verifying that $T_{n}\left(\bar{u}_{\text {orb }}\right)$ is an equivalence.

From the properties of $n$-th derivatives such as $\phi x^{(n)}$ we obtain the upper horizontal arrow in an otherwise obvious commutative square

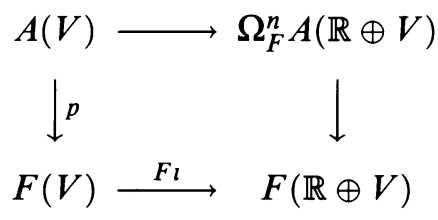

where $\Omega_{F}^{n}$ means: $n$-fold loops staying in a single but arbitrary fiber over $F(\mathbb{R} \oplus V)$. Our assumptions imply that the square is a homotopy pullback square. Iterating this, we obtain the arrow $q$ in a homotopy pullback square

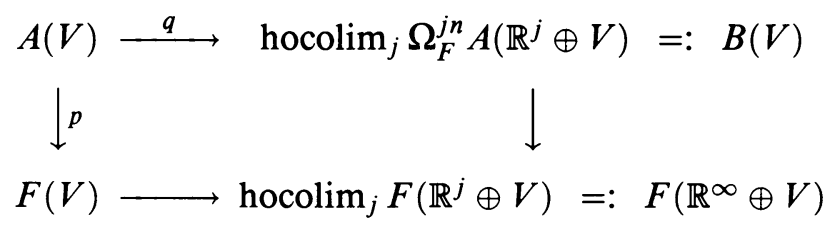

where $F\left(\mathbb{R}^{\infty} \oplus V\right)$ is contractible. It follows that the maps

$$
\begin{aligned}
& (p, q): A(V) \longrightarrow F(V) \times B(V) \\
& \phi x^{(n)}(V, i d) \hookrightarrow B(V) \quad \text { for any } x \in F(V)
\end{aligned}
$$

are homotopy equivalences. These identifications or equivalences respect the $O(n)$-symmetry. It follows that $A_{h O(n)}$ maps by an equivalence to $F \times B_{h O(n)}$, that

$$
\left(T_{n} p, T_{n}\left(q_{h O(n)}\right)\right): T_{n}\left(A_{h O(n)}\right) \longrightarrow T_{n} F \times T_{n}\left(B_{h O(n)}\right)
$$

is an equivalence, and that $T_{n}\left(B_{h O(n)}\right)$ is homogeneous of degree $n$. (Use example 6.4 , and note that $T_{n}$ preserves products up to equivalence.) With 
this knowledge, and the assumption that

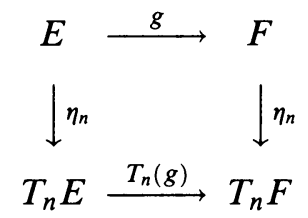

is a homotopy pullback square, it is easy to verify that

$$
T_{n}\left(\bar{u}_{\text {orb }}\right): T_{n}\left(A_{h O(n)}\right) \longrightarrow T_{n} E
$$

satisfies the hypotheses of Corollary 5.13. Hence it is an equivalence. We now define $G$ to be the homotopy pushout of

$$
T_{n}\left(B_{h O(n)}\right) \longleftarrow T_{n}\left(A_{h O(n)}\right) \stackrel{\simeq}{\longrightarrow} T_{n} E
$$

where the left-hand arrow is $T_{n}\left(q_{h O(n)}\right)$. Then $G$ is homogeneous of degree $n$. For $w$, take the composition

$$
E \stackrel{\eta_{n}}{\longrightarrow} T_{n} E \hookrightarrow G
$$

8.2. Theorem [bis]. Moreover, $s$ and $w$ fit into a commutative diagram

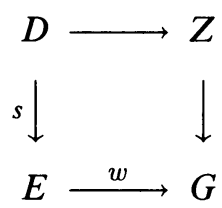

in $\mathscr{E}$, where $Z$ is equivalent to the terminal object * .

Proof. Our construction of $G$ and $w$ so far depends "naturally" on $s$ and $g$. We may write $G(s, g)$ and $w(s, g)$ to indicate the dependence on $s$ and $g$. Form the commutative diagram

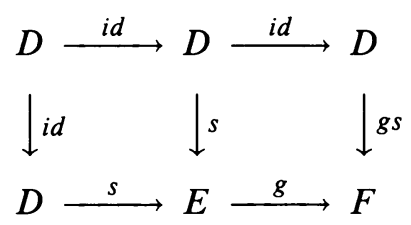

and obtain another commutative diagram

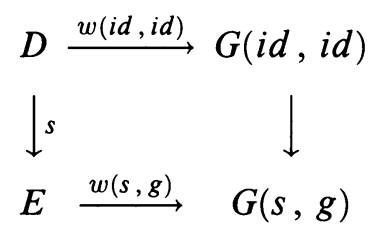

where $G(i d, i d)=: Z$ has to be equivalent to the terminal object.

8.3. Corollary. Let $g: E \rightarrow F$ be a homogeneous morphism of degree $n$ in $\mathscr{E}$, where $n>0$. Assume that $F\left(\mathbb{R}^{\infty}\right)$ is contractible. Then $g$ can be completed to a homotopy pullback square

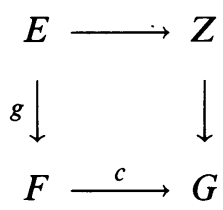


in $\mathscr{E}$, where $G$ is homogeneous of degree $n$ and $Z$ is equivalent to the terminal object. The construction is natural.

Proof. Let $B$ be the homotopy pushout of

$$
F \stackrel{g}{\longrightarrow} E \stackrel{g}{\longrightarrow} F .
$$

This is the fiberwise unreduced suspension of $E$ over $F$, so it comes with a canonical projection map

$$
p: B \longrightarrow F
$$

and two sections

$$
s_{1}, s_{2}: F \longrightarrow B
$$

picking north pole and south pole in each fiber, respectively. (N.b.: We are not assuming that $g$ is a fibration in any sense, although it could be arranged.) The diagram

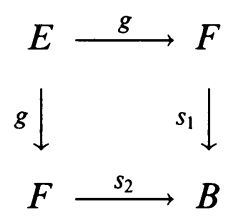

commutes up to a canonical homotopy. It follows from Lemma 7.4 that

$(\$)$

$$
\begin{array}{cc}
T_{n} E \stackrel{T_{n} g}{\longrightarrow} & T_{n} F \\
T_{n} g \downarrow & T_{n} s_{1} \downarrow \\
T_{n} F \stackrel{T_{n} s_{2}}{\longrightarrow} & T_{n} B
\end{array}
$$

(which still commutes up to a canonical homotopy) is a homotopy pullback square. In other words: the canonical homotopy produces an equivalence

$$
T_{n} E \longrightarrow P,
$$

where $P$ is the homotopy pullback of $T_{n} s_{1}$ and $T_{n} s_{2}$. (To prove this, choose some $U$ and $x \in E(U)$. Restriction of $g$ gives a homogeneous morphism of degree $n$ between functors from $\mathscr{L}_{0}\langle U\rangle$ to pointed spaces, with homotopy fiber $D$ say. The homotopy fiber of $T_{n} p$, regarded as a morphism between functors on $\mathscr{J}_{0}\langle U\rangle$, is then equivalent to $T_{n}(\Sigma D)$ which, by Lemma 7.4, is a delooping of $D$. Hence $(\nabla)$ satisfies the hypotheses of Theorem 5.15, hence it is an equivalence.)

Now apply Theorem 8.2 (including [bis]) to the homogeneous morphism $T_{n} p$ with section $T_{n} s_{1}$. The result is a homotopy pullback square

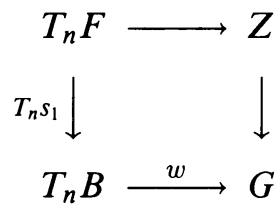

where $Z$ is equivalent to the terminal object and $G$ is homogeneous of degree 
$n$. Plugging this together with previous homotopy pullback squares, we have

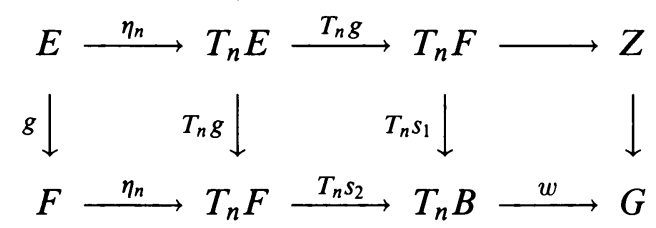

with boundary square

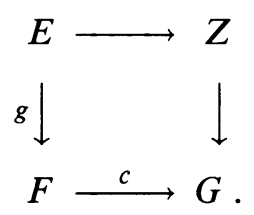

This commutes up to a canonical homotopy and is still a homotopy pullback square. Replacing $Z$ by the mapping cylinder of $E \rightarrow Z$, one can dispose of the homotopy.

Remark. Evidently, $c$ in Corollary 8.3 is in some sense the classifying map for $g$. Is it unique? Write $G(g), Z(g)$ and $c(g)$ for the constructions above, and let

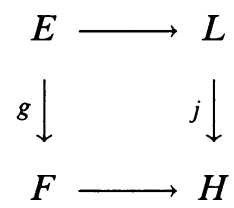

be another homotopy pullback square, where $L$ is equivalent to the terminal object and $H$ is homogeneous of degree $n$. Naturality of our constructions yields yet another commutative square

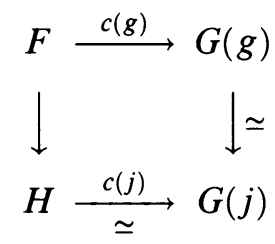

(right-hand vertical arrow induced by the horizontal arrows in $(*)$ ). This establishes uniqueness up to equivalence of the classifying map.

Remark. Note that Corollary 8.3 contains Lemma 7.5 as a special case (take $F=*$ ). We did not use Lemma 7.5 in the proof of Corollary 8.3.

Digression. Homogeneous morphisms of degree $n>0$, as in Definition 8.1, behave in many respects like maps of $C W$-spaces

$$
g: X \longrightarrow Y
$$

whose homotopy fibers are Eilenberg-Mac Lane spaces $K(\pi, n)$. For example, if such a $g$ has a section, and $Y$ is simply connected, then there exists a map $w$ from $X$ to a $K(\pi, n)$ such that

$$
(g, w): X \longrightarrow Y \times K(\pi, n)
$$


is a homotopy equivalence. This is analogous to Theorem 8.2 ; in particular the assumption that $Y$ be simply connected is similar to the assumption that $F\left(\mathbb{R}^{\infty}\right)$ be contractible. Corollary 8.3 is the analogue of the fact that any $g$ as above can be "classified" by a map from $Y$ to a $K(\pi, n+1)$, provided $Y$ is simply connected. (The shift from $n$ to $n+1$ shows that the analogy has a weakness.) When $Y$ is not simply connected, something like "local coefficients" must be used. This is our cue.

We return to the situation of Theorem 8.2, but this time we fix a space $X$ (say a $C W$-space) and consider objects in $\mathscr{E}$ equipped with a reference morphism to the constant functor with value $X$. Denoting this constant functor by $X$ also, we find that we are in the comma category $(\mathscr{E} \downarrow X)$. Suppressing the reference map to $X$, we write $D, E, F$ as usual for objects in $(\mathscr{E} \downarrow X)$. Such an object is homogeneous of degree $n$ over $X$ if the reference map to $X$ is homogeneous of degree $n$.

8.4. Theorem. Let $g: E \rightarrow F$ be homogeneous of degree $n$ in $(\mathscr{E} \downarrow X)$, where $n>0$, and assume that there exists $s: D \rightarrow E$ in $(\mathscr{E} \downarrow X)$ such that $g \cdot s$ is an equivalence. Assume also that the map

$$
F\left(\mathbb{R}^{\infty}\right) \longrightarrow X
$$

(induced by the reference morphism) is a homotopy equivalence. Then there exists $G$ in $(\mathscr{E} \downarrow X)$, homogeneous of degree $n$ over $X$, and a morphism

$$
w: F \longrightarrow G
$$

over $X$ such that

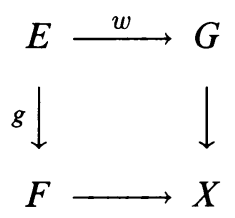

is a homotopy pullback square.

8.4. Theorem [bis]. Moreover, $s$ and $w$ fit into a commutative square

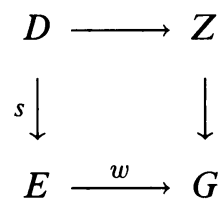

in $(\mathscr{E} \downarrow X)$, where $Z$ is equivalent to the terminal object in $(\mathscr{E} \downarrow X)$.

8.5. Corollary. Let $g: E \rightarrow F$ be a homogeneous morphism of degree $n$ in $(\mathscr{E} \downarrow X)$, where $n>0$. Assume that $F\left(\mathbb{R}^{\infty}\right) \rightarrow X$ is a homotopy equivalence. Then $g$ can be completed to a homotopy pullback square

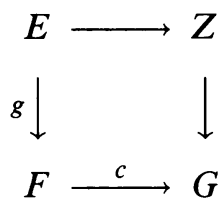


in $(\mathscr{E} \downarrow X)$, where $G$ is homogeneous of degree $n$ over $X$ and $Z$ is equivalent to the terminal object in $(\mathscr{E} \downarrow X)$. The construction is natural.

Notation for the proof of Theorem 8.4. For any $C$ in $(\mathscr{E} \downarrow X)$ and any map $q$ from a $C W$-space $Y$ to $X$, we define $q$ 「C in $\mathscr{E}$ by

$$
\left(q\ulcorner C)(W):=\text { hofiber }\left[C(W)^{Y} \rightarrow X^{Y}\right]\right.
$$

(homotopy fiber over the base point $q \in X^{Y}$ ). The exponential notation means mapping spaces. Think of $q$ 队 $C$ as "lifts of $q$ to $C$ ".

Sketch proof of Theorem 8.4. We will deduce this from Theorem 8.2, making strong use of naturality and simplicial machinery. For any singular simplex

$$
q: \Delta^{k} \longrightarrow X \text {, }
$$

the diagram

$$
q \text { } D \stackrel{q \longmapsto s}{\longrightarrow} q \longmapsto E \stackrel{q » g}{\longrightarrow} q \longmapsto F
$$

in $\mathscr{E}$ has all the properties required in Theorem 8.2 ; in particular $(q \longmapsto F)\left(\mathbb{R}^{\infty}\right)$ is contractible. Therefore

$$
w=w_{q}: q \longmapsto E \longrightarrow G_{q}
$$

is defined such that $\left(q \longmapsto g, w_{q}\right)$ is an equivalence. Define $\widetilde{E}$ in $(\mathscr{E} \downarrow X)$ by

$$
\widetilde{E}(W):=\left|[k] \mapsto \coprod_{q: \Delta^{k} \rightarrow X}(q \longmapsto E)(W)\right|
$$

(vertical bars for geometric realization, of a simplicial space). The evaluation maps

$$
\triangle^{k} \times(q \longmapsto E) \longrightarrow E \quad \text { for } q: \Delta^{k} \rightarrow X
$$

give us an equivalence $\widetilde{E} \rightarrow E$. Define similarly $\widetilde{G}$ by

$$
\widetilde{G}(W):=\mid[k] \mapsto \coprod_{q: \Delta^{k} \rightarrow X}(q\ulcorner G)(W) \mid
$$

so that the $w_{q}$ define a map $\widetilde{E} \rightarrow \widetilde{G}$. Let $G$ be the homotopy pushout of

$$
E \longleftarrow \widetilde{E} \longrightarrow \widetilde{G}
$$

and let $w: E \rightarrow G$ be the inclusion. It remains to understand how and why $G$ is an object over $X$. Briefly, it works like this: There is the reference morphism from $E$ to $X$, and there is another fairly obvious morphism from $\widetilde{G}$ to $X$ (via the geometric realization of the singular simplicial set of $X$ ). Finally there is a more subtle evaluation morphism

$$
\widetilde{E} \times[0,1] \longrightarrow X,
$$

compatible with the previous two.

8.6. Remark. Notice that Theorem 8.4 and Corollary 8.5 are sufficiently general in the following sense. For arbitrary $F$ in $\mathscr{E}$, let

$$
X:=F\left(\mathbb{R}^{\infty}\right)=\left(T_{0} F\right)(0) .
$$


Then, although there is no obvious reference morphism from $F$ to $X$, there is an obvious diagram

$$
F \stackrel{\eta_{0}}{\longrightarrow} T_{0} F \stackrel{\simeq}{\longleftarrow}
$$

and we can replace $F$ by the homotopy pullback $F^{\sharp}$ of this diagram. We have

$$
F \stackrel{\simeq}{\simeq} F^{\sharp} \longrightarrow X
$$

and the reference morphism from $F^{\sharp}$ to $X$ is such that

$$
F^{\sharp}\left(\mathbb{R}^{\infty}\right) \stackrel{\simeq}{\longrightarrow} X .
$$

Alternatively, Corollary 8.5 (for example) can be rephrased in the following way. Any $g: E \rightarrow F$ in $\mathscr{E}$ which is homogeneous of degree $n$ fits into a homotopy pullback square

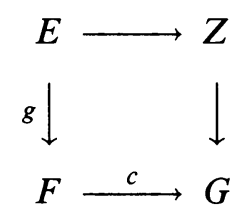

where $Z$ is polynomial of degree 0 , all morphisms are approximations of order 0 , and $G$ is locally homogeneous of degree $n$. "Locally homogeneous of degree $n$ " means that the map $\eta_{0}$ from $G$ to $T_{0} G$ is homogeneous of degree $n$. As before, the construction of ( from the homogeneous morphism $g$ is natural, and naturality implies a certain uniqueness.

To make Corollary 8.5 a little more explicit, we should now classify diagrams

$$
Z \stackrel{s}{\longrightarrow} G \stackrel{t}{\longrightarrow} X
$$

in $(\mathscr{E} \downarrow X)$, where $t s$ is an equivalence and $t$ is homogeneous of degree $n$. Classification can be up to equivalence (over $X$ ), and therefore we may assume without loss of generality that $Z=X$ and $t s=i d_{X}$. We now have a parametrized version, with parameter space $X$, of the classification problem solved in Theorem 7.3. Much as in the proof of Theorem 8.4, the parametrized problem can be reduced to the unparametrized one. However, I shall omit the details of the argument and state the result only.

\subsection{Notation.}

(1) If $A$ and $B$ are spaces and

$$
p: A \rightarrow B
$$

is a fibration, write

$$
A=\int_{B} p^{-1}(x) d x .
$$

This suggests that $A$ is the "integral" of a parametrized family of spaces $p^{-1}(x)$, parametrized by the points $x \in B$. It is convenient notation if the fibers of the fibration have "names", and the total space (here $A$ ) has no "name".

(2) For arbitrary $E$ in $\mathscr{E}$ let $h_{E, n}$ be the "homology theory" taking a pointed $O(n)-C W$-space $Y$ to

$$
\underset{k}{\operatorname{hocolim}} \int_{E\left(\mathbb{R}^{k}\right)} \Omega^{\infty}\left[\left(Y \wedge \boldsymbol{\Theta}_{x}\right)_{h O(n)}\right] d x
$$


where $\boldsymbol{\Theta}_{x}$ is short for $\boldsymbol{\Theta}^{\sharp} E_{x}^{(n)}$, as defined in and around Theorem 5.15 (cf. also section 3). Let $O(n)$ act on $Y \wedge \boldsymbol{\Theta}_{x}$ by the diagonal action.

Note that $h_{E, n}(Y)$ is a retractive space over $h_{E, n}(*) \cong E\left(\mathbb{R}^{\infty}\right)$. The functor $h_{E, n}$ takes homotopy pushout squares to homotopy pullback squares. (A square of pointed $O(n)-C W$-spaces and pointed $O(n)$-maps is a homotopy pushout square if the underlying square of $C W$-spaces is.)

8.8. Rough statement. Let $G$ be homogeneous of degree $n$ over $X$, with section $s: X \rightarrow G$, where $n>0$. Then $G$ is equivalent, rel. $X$, to the functor

$$
V \longmapsto h_{G, n}\left((n V)^{c}\right) \text {. }
$$

Continuing in this vein, we note that the homotopy pullback square in Corollary 8.5 (or ( in Remark 8.6) gives rise to a homotopy pullback square of homology theories

such that
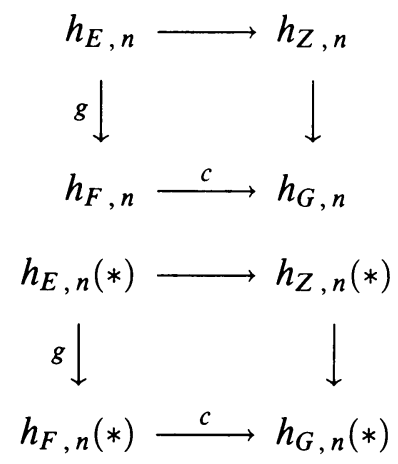

consists entirely of homotopy equivalences. In this situation it follows from general considerations about homology theories that $h_{G, n}$ is determined by

$$
g: h_{E, n} \longrightarrow h_{F, n}
$$

(up to a chain of natural homotopy equivalences). Namely, up to such a chain, $h_{G, n}(Y)$ is the homotopy pullback of

$$
\begin{gathered}
h_{F, n}(*) \\
\downarrow s \\
h_{E, n}(\Sigma Y) \stackrel{g}{\longrightarrow} h_{F, n}(\Sigma Y)
\end{gathered}
$$

for any pointed $O(n)-C W$-space $Y$. The vertical arrow $s$ is the standard section.

\section{THE TAYLOR TOWER}

The constructions of section 6 produce, for each object $E$ in $\mathscr{E}$, a diagram

$$
\cdots \rightarrow T_{n+1} E \stackrel{r_{n+1}}{\longrightarrow} T_{n} E \stackrel{r_{n}}{\longrightarrow} T_{n-1} E \stackrel{r_{n-1}}{\longrightarrow} \cdots \quad \cdots \stackrel{r_{1}}{\longrightarrow} T_{0} E,
$$

the Taylor tower of $E$. The morphisms $r_{n}$ satisfy

$$
r_{n} \eta_{n}=\eta_{n-1}: E \longrightarrow T_{n-1} E,
$$

and they can be described explicitly as forgetful morphisms. Namely, $T_{n} E$ is a homotopy colimit of objects $\left(\tau_{n}\right)^{k}(E)$ (where $k$ "tends to infinity"), and there 
are evident forgetful morphisms from $\tau_{n}$ to $\tau_{n-1}$. Alternatively, we could use the (so-called) universal property of

$$
\eta_{n}: E \longrightarrow T_{n} E
$$

which should give us a unique map $r_{n}$ from $T_{n} E$ to $T_{n-1} E$ such that $r_{n} \eta_{n}=$ $\eta_{n-1}$. However, since the universal property in question only works "up to equivalence", this is not quite so neat. We are fortunate to have an explicit $r_{n}$.

We note that $r_{n}$ is an approximation of order $n-1$, since $\eta_{n}$ and $\eta_{n-1}$ are (by Theorem 6.3 [bis]). This implies that $T_{n-1} r_{n}$ is also an approximation of order $n-1$, hence an equivalence by Theorem 5.15. Thus $r_{n}$ is homogeneous of degree $n$.

When $E$ belongs to $\mathscr{E}_{0}$, then the $T_{n} E$ are also objects of $\mathscr{E}_{0}$ for $n \geq 0$, and the homotopy fiber of

$$
r_{n}: T_{n} F \longrightarrow T_{n-1} F
$$

(over the base point) is defined for $n>0$. It is of course homogeneous of degree $n$, and the classification Theorem 7.3 tells us the following.

9.1. Theorem. For $E$ in $\mathscr{E}_{0}$, any $n>0$, any $V$ in $\mathscr{J}$, there is a fibration sequence up to homotopy

$$
\Omega^{\infty}\left[\left((n V)^{c} \wedge \boldsymbol{\theta}\right)_{h O(n)}\right] \longrightarrow\left(T_{n} E\right)(V) \stackrel{r_{n}}{\longrightarrow}\left(T_{n-1} E\right)(V)
$$

where $\boldsymbol{\theta}$ depends on $E$ and $n$ only: $\boldsymbol{\theta}=\boldsymbol{\theta}^{\sharp} E^{(n)}$, the spectrum with action of $O(n)$ defined in section 3.

We can get a stronger (if less explicit) result by using Corollary 8.5. This gives us a homotopy pullback square

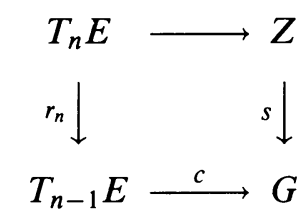

where $Z$ is of degree 0 , all arrows are approximations of order 0 , and $G$ is locally homogeneous of degree $n$ (cf. Remark 8.6). By the rough statement 8.8 and sequel, $G$ has the form or is equivalent to

$$
V \longmapsto h_{E, n}\left(\Sigma(n V)^{c}\right)
$$

(notation of 8.7 ). Further unravelling is left to the reader.

\section{MoRe EXAMPLeS}

10.1. Example. Let $F$ in $\mathscr{E}_{0}$ be given by $F(V)=O\left(\mathbb{R}^{\infty} \oplus V\right) / O(V)$. Then $T_{0} F$ vanishes, and $\boldsymbol{\theta} F^{(1)}$ is homotopy equivalent to $\mathbf{S}^{0}$, with the trivial action of $O(1)$. (Cf. Example 2.7, which is essentially the same example, up to "extension" by a functor of degree 0 .) Hence we have

$$
\eta_{1}: O\left(\mathbb{R}^{\infty} \oplus V\right) / O(V) \longrightarrow\left(T_{1} F\right)(V) \simeq Q\left[\left(V^{c}\right)_{h O(1)}\right]
$$

Here $O(1)$ acts on $V^{c}$ by $v \mapsto-v$ for $v \in V$, so we can rewrite the codomain:

$$
Q\left[\left(V^{c}\right)_{h O(1)}\right] \cong Q\left(P\left(\mathbb{R}^{\infty} \oplus V\right) / P(V)\right),
$$


where $P$ denotes projective spaces. Since $\eta_{1}$ is an approximation of order 1 , the map of horizontal homotopy fibers in

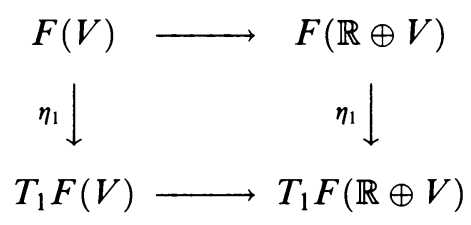

is, up to various homotopy equivalences, the stabilization map

$$
V^{c} \hookrightarrow Q\left(V^{c}\right)
$$

Applying all this with $V=\mathbb{R}^{k}$, for arbitrary $k$, one finds that

$$
\eta_{1}: F(0)=O(\infty) \quad \longrightarrow \quad\left(T_{1} F\right)(0) \simeq Q\left(\mathbb{R} P_{+}^{\infty}\right)
$$

maps the nonzero class in $H^{k}\left(\mathbb{R} P^{\infty} ; \mathbb{Z} / 2\right)$ (spectrum cohomology of the codomain) to the class $\Omega w_{k+1}$ in $H^{k}(O(\infty) ; \mathbb{Z} / 2)$ ), for all $\mathrm{k}$. Here $w_{k+1}$ is the Stiefel-Whitney class.

10.2. Example. Let $E$ be given by

$$
E(V)=U\left(\mathbb{C}^{\infty} \oplus V\right) / U(V)
$$

for finite dimensional complex vector spaces $V$ with a hermitian positive definite inner product. (This is unitary calculus.) One finds that

$$
\mathbf{\theta} E^{(1)} \simeq \mathbf{S}^{-1} \simeq \Omega \mathbf{S}^{0}
$$

with the trivial action of the unitary group $U(1)$. As in the previous example,

$$
\eta_{1}: E(V) \longrightarrow\left(T_{1} E\right)(V)
$$

takes the form

$$
U\left(\mathbb{C}^{\infty} \oplus V\right) / U(V) \longrightarrow \Omega Q\left(P\left(\mathbb{C}^{\infty} \oplus V\right) / P(V)\right) ;
$$

here $P(-)$ denotes complex projective spaces. (The $\Omega$ comes from $(\checkmark)$.) For $V=0$ this is

$$
s: U(\infty) \longrightarrow \Omega Q\left(B U(1)_{+}\right)
$$

and the adjoint $\Sigma^{\infty+1} U \rightarrow \Sigma^{\infty} B U(1)$ maps one of the generators of $H^{2 k}(B U(1) ; \mathbb{Z})$ to

$$
\Omega c_{k} \in H^{2 k-1}(U ; \mathbb{Z})
$$

(the looped Chern class), for all $k>0$. Hence $s$ induces a surjection in integral cohomology, and an isomorphism in rational cohomology. Equivalently, $s$ induces a surjection in integral cohomology, with kernel equal to the torsion subgroup.

An interesting consequence is that the map $s$ has a homotopy left inverse. A natural candidate for such a left inverse is $p=\Omega q$, where $q$ is the composition

$$
Q\left(B U(1)_{+}\right) \hookrightarrow Q\left(B U_{+}\right) \stackrel{\mu}{\longrightarrow} B U .
$$

Here $\mu$ uses the infinite loop space structure on $B U$. By [Se1], the map $p$ has a homotopy right inverse, say $s_{1}$, so that $p s_{1} \simeq i d$. (In fact according to [Se1] the map $q$ has a homotopy right inverse.) Now $s_{1}$ must induce a surjection in 
integral cohomology, with kernel equal to the torsion subgroup, just like $s$. It follows that $p s$ induces an isomorphism in cohomology, like $p s_{1}$.

10.3. Example. Let $F(V)=B O(V)$. Then

$$
\eta_{2}: F(V) \rightarrow T_{2} F(V)
$$

is a rational homotopy equivalence for every $V \neq 0$. (This was pointed out by the referee.) Much of this has been proved in 2.7: We saw that $F^{(3)}(V)$ is always rationally homotopy equivalent to a point. It follows (Proposition 5.3) that $\Omega F$ is rationally polynomial of degree 2 , hence that the canonical morphism $\Omega F \rightarrow T_{2}(\Omega F)$ is an equivalence. Since $T_{2}$ commutes with $\Omega$ up to equivalence (by construction), we may conclude that

$$
F(V) \longrightarrow T_{2} F(V)
$$

is a homotopy equivalence provided $T_{2} F(V)$ is connected. For $V \neq 0$, this is easily proved by a two-step induction using e.g. Theorem 9.1 and the calculation of $\boldsymbol{\theta} F^{(1)}$ and $\boldsymbol{\theta} F^{(2)}$ in 2.7 .

10.4. Example. Here we let $F(V)=O\left(\mathbb{R}^{\infty} \oplus V\right) / O(V)$ as in 10.1. Complexification gives

$$
F(V) \longrightarrow E\left(V \otimes_{\mathbb{R}} \mathbb{C}\right) \stackrel{\eta_{1}}{\longrightarrow} T_{1} E\left(V \otimes_{\mathbb{R}} \mathbb{C}\right)
$$

where $E$ is from Example 10.2, and $T_{1}$ is the first Taylor approximation in unitary calculus. Now it is clear that the continuous functor

$$
V \mapsto T_{1} E\left(V \otimes_{\mathbb{R}} \mathbb{C}\right)
$$

on $\mathcal{J}$ is polynomial of degree 2 . Hence we have a factorization

$$
F(V) \longrightarrow T_{2} F(V) \longrightarrow T_{1} E\left(V \otimes_{\mathbb{R}} \mathbb{C}\right)
$$

Specializing to $V=0$, we see that the looped integral Pontryagin classes in the cohomology of $O(\infty)=F(0)$ lift to $H^{*}\left(T_{2} F(0) ; \mathbb{Z}\right)$.

10.5. Example. Let $D$ in $\mathscr{E}_{0}$ be defined by

$$
D(V)=B \mathscr{G}(S(V))
$$

where $S(V)$ is the unit sphere in $V$ and $\mathscr{G}(S(V))$ is the grouplike monoid of its homotopy automorphisms. It is proved in [WW1, 3.8] that

$$
\mathbf{\theta} D^{(1)} \simeq \mathbf{S}^{0}
$$

with the trivial action of $O(1)$. Hence the map of horizontal homotopy fibers in

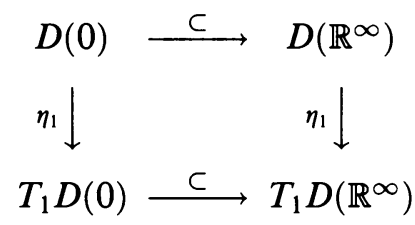

becomes (up to homotopy equivalences)

$$
\mathscr{G} \longrightarrow Q\left(\mathbb{R} P_{+}^{\infty}\right)
$$

where $\mathscr{G} \subset Q S^{0}$ consists of the components of degree \pm 1 . From the geometric description of $(\diamond)$ given in [WW1], which is easily related to the one used here, 
one obtains the following. Composition of $(\diamond)$ with the transfer from $Q\left(\mathbb{R} P_{+}^{\infty}\right)$ to $Q\left(S_{+}^{\infty}\right) \simeq Q S^{0}$ gives

$$
\text { inclusion }-c_{1}: \mathscr{G} \longrightarrow Q S^{0}
$$

where $c_{1}$ is the constant map with value 1 . In particular, the map $(\diamond)$ has a homotopy left inverse.

So far I have not found a much more direct argument to prove this fact.

10.6. Example. Let us finish the calculation of $\boldsymbol{\theta} F^{(3)}$ in Example 2.7, where $F(V)=B O(V)$. We only have to show that $\mathbf{8} F^{(3)}$ is not contractible. (Again I am indebted to John Klein for help with the argument.) Let $A(V)=V^{c}$. There is a fibration sequence up to homotopy

$$
A(V) \longrightarrow F(V) \hookrightarrow F(\mathbb{R} \oplus V) .
$$

Hence we only have to show that $\boldsymbol{\theta} A^{(3)}$ is not contractible. Let $B(V)$ be the homotopy fiber of the stabilization map

$$
A(V)=V^{c} \longrightarrow Q\left(V^{c}\right)
$$

where $Q=\Omega^{\infty} \Sigma^{\infty}$. Now we only have to show that $\boldsymbol{\theta} B^{(3)}$ is not contractible. Let

$$
G(V)=Q\left[(3 V)^{c}{ }_{h K}\right],
$$

where $K$ is the symmetric group on three letters (and $h K$ denotes the Borel construction in the pointed category). Note that $G$ is homogeneous of degree 3 by Theorem 7.3 and some inspection. For $V \neq 0$, think of $(3 V)^{c} h K$ as the appropriate factor in the (stable) Snaith splitting [Sn] of $Q\left(V^{c}\right)$. Projection to this factor then gives us a natural transformation

$$
\zeta: B(V \oplus \mathbb{R}) \longrightarrow \Omega G(V \oplus \mathbb{R}) .
$$

By the universal property of $T_{3} B$, this must factor (up to equivalence) through

$$
\eta_{3}: B(V \oplus \mathbb{R}) \longrightarrow T_{3} B(V \oplus \mathbb{R})
$$

since $G$ has degree 3 . Let us show that it does not factor through

$$
\eta_{2}: B(V \oplus \mathbb{R}) \longrightarrow T_{2} B(V \oplus \mathbb{R})
$$

by considering the case $V=0$. Indeed, $T_{1} B(\mathbb{R})$ vanishes by construction, and therefore

$$
T_{2} B(\mathbb{R}) \simeq \Omega^{\infty}\left(\left(S^{2} \wedge \mathbf{8}\right)_{h O(2)}\right) \quad\left(\mathbf{\theta}=\mathbf{8} B^{(2)} \simeq \mathbf{8} A^{(2)}\right)
$$

by Theorem 9.1 . From the natural fibration sequence up to homotopy (!) and the calculation of $\boldsymbol{\theta} F^{(2)}$ in Example 2.7, we obtain

$$
S^{2} \wedge \boldsymbol{O} \simeq \mathbf{S}^{1} \vee \mathbf{S}^{2}
$$

Then, combining (!!) and (!!!), we find that $\pi_{3}\left(T_{2} B(\mathbb{R})\right)$ has no 3-torsion. But $\zeta$ detects the 3 -torsion in $\pi_{3}(B(\mathbb{R}))=\pi_{3}\left(\Omega Q S^{1}\right)$. (Otherwise the stable Snaith splitting of $Q S^{1}$ would not be a splitting.) Hence $T_{3} B$ is sufficiently different from $T_{2} B$, and $\boldsymbol{\theta} B^{(3)}$ cannot be contractible.

\section{APPENDIX: COFIBRANT OBJECTS}

A.1. Conventions. In this appendix, the category $\mathcal{J}$ has only the following objects: the vector spaces $\mathbb{R}^{k}$ for $k \geq 0$. The space of morphisms from $\mathbb{R}^{j}$ 
to $\mathbb{R}^{k}$ is, as usual, the Stiefel manifold $\operatorname{mor}\left(\mathbb{R}^{j}, \mathbb{R}^{k}\right)$. Furthermore, $\mathscr{E}$ is the category of continuous functors from $\mathscr{J}$ to spaces (say, spaces having the homotopy type of a $C W$-space). As before, a morphism $g: E \rightarrow F$ in $\mathscr{E}$ is an equivalence if $g_{V}: E(V) \rightarrow F(V)$ is a homotopy equivalence for all $V$. An object $E$ in $\mathscr{E}$ is cofibrant if, for any diagram

$$
E \stackrel{c}{\longrightarrow} F \stackrel{e}{\longleftarrow} \hat{F}
$$

where $e$ is an equivalence, there exists a morphism

$$
\hat{c}: E \longrightarrow \hat{F}
$$

and a natural homotopy $h$ from $e \cdot \hat{c}$ to $c$.

As a category, the "new" $\mathcal{J}$ is of course equivalent to the old one. It follows that the new $\mathscr{E}$ is equivalent to the old one.

A.2. Definition. A $C W$-object in $\mathscr{E}$ is an object $E$ together with a filtration by subobjects,

$$
\varnothing=E_{-1} \subset E_{0} \subset E_{1} \subset E_{2} \subset E_{3} \subset \ldots
$$

such that the following holds.

(1) $E(V)=\bigcup E_{i}(V)=$ colim $E_{i}(V)$ with the colimit topology, for all $V$ in $\mathcal{J}$.

(2) For each $i \geq 0$, the object $E_{i}$ is isomorphic rel $E_{i-1}$ to the pushout of a diagram

$$
E_{i-1} \longleftarrow S^{i-1} \times K \hookrightarrow D^{i} \times K
$$

where $K=\amalg_{\lambda} \operatorname{mor}\left(V_{\lambda},-\right)$ is a coproduct of representable objects in $\mathscr{E}$.

A.3. Observation. Any $C W$-object $E$ in $\mathscr{E}$ is cofibrant.

Proof. Given

$$
E \stackrel{c}{\longrightarrow} F \stackrel{e}{\longleftarrow} \hat{F}
$$

where $e$ is an equivalence, define $\hat{c}: E \rightarrow \hat{F}$ and the homotopy $h$ from $e \cdot \hat{c}$ to $c$ by induction on skeletons, using the obvious fact that representable objects in $\mathscr{E}$ are cofibrant.

A.4. Observation. For any object $F$ in $\mathscr{E}$, there exists a $C W$-object $F^{\diamond}$ and an equivalence $e: F^{\diamond} \rightarrow F$.

Proof. Assume that the $i$-skeleton $F_{i}^{\diamond}$ of $F^{\diamond}$ and the restriction of $e$ to $F_{i}^{\diamond}$ have already been constructed. Form the set of all triples $\left(V_{\lambda}, f_{\lambda}, g_{\lambda}\right)$ where $V_{\lambda}$ in $\mathscr{J}$ and $f_{\lambda}, g_{\lambda}$ fit into a commutative square

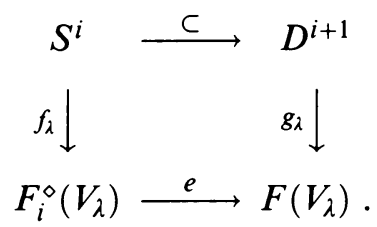

We construct $F_{i+1}^{\diamond}$ as the homotopy pushout of

$$
F_{i}^{\diamond} \stackrel{\chi}{\longleftarrow} \coprod_{\lambda} S^{i} \times \operatorname{mor}\left(V_{\lambda},-\right) \hookrightarrow \coprod_{\lambda} D^{i} \times \operatorname{mor}\left(V_{\lambda},-\right)
$$


where $\chi$ agrees with $f_{\lambda}$ on

$$
S^{i} \cong S^{i} \times\{i d\} \subset S^{i} \times \operatorname{mor}\left(V_{\lambda}, V_{\lambda}\right) .
$$

(Always remember the Yoneda lemma.) Similarly, we construct the extension of $e$ to $F_{i+1}^{\diamond}$ so that it agrees with $g_{\lambda}$ on

$$
D^{i+1} \cong D^{i+1} \times\{i d\} \subset D^{i+1} \times \operatorname{mor}\left(V_{\lambda}, V_{\lambda}\right)
$$

By induction on $i$, the restriction

$$
e: F_{i}^{\diamond}(W) \longrightarrow F(W)
$$

is $i$-connected for any $i$, any $W$.

Remark. The construction of $F^{\diamond}$, and of the equivalence to $F$, is natural. When $F$ belongs to $\mathscr{E}_{0}$, which means that a base point in $F(0)$ has been selected, then there is also a preferred base point in $F^{\diamond}(0)$. The construction $F \mapsto F^{\diamond}$ can also be applied to a continuous functor from $\mathcal{J}$ to quite arbitrary spaces (which need not have the homotopy type of $C W$-spaces). In such a case

$$
F^{\diamond}(W) \stackrel{e}{\longrightarrow} F(W)
$$

will be a weak homotopy equivalence, for all $W$.

Even more generally, it is not necessary to insist on a functor $F$ whose values $F(V)$ are spaces; it is sufficient to know what the continuous maps from compact spaces $K$ to $F(V)$ are. The functor

$$
V \longrightarrow \operatorname{DIFF}^{b}(M \times V)
$$

(cf. Example 3.2) is of this type.

\section{ACKNOWLEDGMENT}

It is a pleasure to thank Tom Goodwillie, John Klein and Bruce Williams for conversations and encouragement. Much of this paper was conceived at Aarhus University, Denmark, but the final touches were added during an eight-month visit to the SFB 343, Bielefeld University.

\section{REFERENCES}

[Ad] J.F. Adams, Stable homotopy and generalised homology, Univ. of Chicago Press, Chicago IL, 1974.

[AnH] D.R. Anderson and W.-C. Hsiang, The functors $K_{-i}$ and pseudo-isotopies of polyhedra, Ann. of Math. 105 (1977), 201-223.

[BK] A.K. Bousfield and D.M. Kan, Homotopy limits, completions, and localizations, Lecture Notes in Math., vol. 304, Springer-Verlag, New York, 1972.

[DWW] W. Dwyer, M. Weiss and B. Williams, A parametrized index theorem for the algebraic $K$-theory Euler class, Preprint 63 pp., 1995.

[EM] S. Eilenberg and J.C. Moore, Homology and fibrations I, Comment. Math. Helv. 40 (1966), 199-236.

[EMaL] S. Eilenberg and S. Mac Lane, On the groups $K(\pi, n)$, Ann. of Math. 60 (1954), 49-139.

[Go1] T.G. Goodwillie, Calculus I: The first derivative of pseudisotopy theory, K-Theory 4 (1990), $1-27$.

[Go2] _ Calculus II: Analytic functors, K-Theory 5 (1992), 295-332. 
[Ha] A. Hatcher, Higher simple homotopy theory, Ann. of Math. 102 (1975), 101-137.

[Ja] I.M. James, Reduced product spaces, Ann. of Math. 62 (1955), 170-197.

[KP] D.S. Kahn and S.B. Priddy, Applications of the transfer to stable homotopy theory, Bull. Amer. Math. Soc. 78 (1972), 981-987.

[MaL] S. Mac Lane, Categories for the working mathematician, Springer-Verlag, New York, 1971.

[Ma] J.P. May, $E_{\infty}$ Ring spaces and $E_{\infty}$ ring spectra, Lecture Notes in Math., vol. 577, Springer-Verlag, New York, 1977.

[Se1] G.B. Segal, The stable homotopy of complex projective space, Quart. J. Math. Oxford (2) 24 (1973), 1-5.

[Se2] Classifying spaces and spectral sequences, Inst. Hautes Etudes Sci. Publ. Math. 34 (1968), 105-112.

[Sn] V.P. Snaith, A stable decomposition of $\Omega^{n} S^{n} X$, J. London Math. Soc. (2) 7 (1974), 577-583.

[WaMA] F. Waldhausen, Algebraic K-theory of spaces, a manifold approach, Canad. Math. Soc. Conf. Proc., vol. 2, part 1, Amer. Math. Soc., Providence, R.I., 1982, pp. 141-186.

[We] M. Weiss, Pinching and concordance theory, J. Differential Geom. 38 (1993), 387-416.

[WW1] M. Weiss and B. Williams, Automorphisms of manifolds and algebraic K-theory: I, KTheory 1 (1988), 575-626.

[WW2] _ Automorphisms of manifolds and algebraic K-theory: II, J. Pure Appl. Algebra 62 (1989), 47-107.

[WW3] _ Automorphisms of manifolds and algebraic K-theory: Finale, preprint 1994.

Department of Mathematics, University of Michigan, ANn Arbor, Michigan 481091003

E-mail address: msweiss@math.lsa.umich.edu 